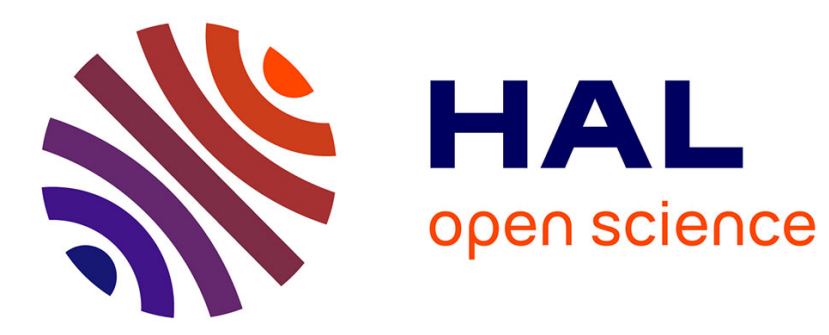

\title{
Stand-Alone Extraction of Cyclostationary Broadband Components from Aeroacoustic Signals
}

Jérôme Antoni, Quentin Leclère, Alice Dinsenmeyer, Emmanuel Julliard, Simon Bouley, Christophe Picard, Pieter Sijtsma

\section{To cite this version:}

Jérôme Antoni, Quentin Leclère, Alice Dinsenmeyer, Emmanuel Julliard, Simon Bouley, et al.. StandAlone Extraction of Cyclostationary Broadband Components from Aeroacoustic Signals. AIAA Journal, inPress, 10.2514/1.J060289 . hal-03352598

\section{HAL Id: hal-03352598 \\ https://hal.science/hal-03352598}

Submitted on 23 Sep 2021

HAL is a multi-disciplinary open access archive for the deposit and dissemination of scientific research documents, whether they are published or not. The documents may come from teaching and research institutions in France or abroad, or from public or private research centers.
L'archive ouverte pluridisciplinaire $\mathbf{H A L}$, est destinée au dépôt et à la diffusion de documents scientifiques de niveau recherche, publiés ou non, émanant des établissements d'enseignement et de recherche français ou étrangers, des laboratoires publics ou privés. 


\title{
Stand-Alone Extraction of Cyclostationary Broadband Components from Aeroacoustic Signals
}

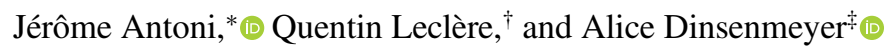 \\ Univ Lyon, INSA Lyon, LVA, EA677, 69621 Villeurbanne, France \\ Emmanuel Julliard $\stackrel{\S}{-}$ \\ Airbus Operations S.A.S, 31300 Toulouse, France \\ Simon BouleyI and Christophe Picard" \\ MicrodB, F-69134 Écully, France \\ and \\ Pieter Sijtsma立 \\ PSA3, 9493 TE De Punt, The Netherlands \\ https://doi.org/10.2514/1.J060289
}

\begin{abstract}
Aeroacoustic signals, as typically returned by recordings of pressure fluctuations generated by rotating machines, often exhibit a strong contribution of broadband components. Separate analysis of the various sources of broadband noise is important from an engineering point of view, as they relate to different physical mechanisms. It is yet technically difficult to achieve, as the corresponding signals overlap in both time and frequency. A solution is proposed based on the angle-time cyclostationary property of broadband noise produced by rotating sources. Cyclostationary broadband components can thus be extracted, resynthesized in the time domain, and analyzed individually as if they were measured alone with all other interfering sources switched off. A special effort is made to render the methodology as stand-alone as possible by providing criteria for automatically setting up the leading parameters. The methodology is illustrated in counter-rotating open rotor data, where cyclostationary broadband contributions from the rear and front rotors and their interaction are individually extracted.
\end{abstract}

\begin{tabular}{|c|c|c|}
\hline \multicolumn{3}{|r|}{ Nomenclature } \\
\hline $\mathcal{A}$ & $=$ & set of discrete cyclic orders \\
\hline $\mathcal{A}^{*}$ & & set $\mathcal{A}$ with zero order removed \\
\hline$f$ & & frequency, $\mathrm{Hz}$ \\
\hline$I$ & $=$ & $\begin{array}{l}\text { number of time instants in short-time (angular) } \\
\text { Fourier transform }\end{array}$ \\
\hline$j$ & $=$ & imaginary unit \\
\hline$K$ & & $\begin{array}{l}\text { cardinal of set } \mathcal{A}^{*} \text { (i.e., number of cyclic orders } \\
\text { used in cyclic regression) }\end{array}$ \\
\hline$L$ & $=$ & signal length (samples) \\
\hline$M$ & $=$ & number of sensors \\
\hline$N$ & $=$ & $\begin{array}{l}\text { matrix of short-time Fourier coefficients of signals } \\
n_{m}(t) \text {, where } m \text { is equal to } 1, \ldots, M\end{array}$ \\
\hline$N_{w}$ & $=$ & kernel length (samples) \\
\hline$n(t)$ & $=$ & stationary part of aeroacoustic signal \\
\hline$p(t)$ & $=$ & tonal part of aeroacoustic signal \\
\hline $\boldsymbol{R}$ & $=$ & $\begin{array}{l}\text { matrix of short-time Fourier coefficients of signals } \\
r_{m}(t), \text { where } m \text { is equal to } 1, \ldots, M\end{array}$ \\
\hline$R_{m i}\left(f_{k}\right)$ & $=$ & short-time Fourier transform of signal $r(t)$ \\
\hline$R_{r r^{\prime}}(\theta, t)$ & $=$ & $\begin{array}{l}\text { angle-time cross-correlation function between } \\
\text { signals } r(t) \text { and } r^{\prime}(t)\end{array}$ \\
\hline$r(t)$ & $=$ & $\begin{array}{l}\text { angle-time cyclostationary part of aeroacoustic } \\
\text { signal }\end{array}$ \\
\hline
\end{tabular}

Received 30 October 2020; revision received 7 August 2021; accepted for publication 9 August 2021; published online 21 September 2021. Copyright (C) 2021 by the American Institute of Aeronautics and Astronautics, Inc. All rights reserved. All requests for copying and permission to reprint should be submitted to CCC at www.copyright.com; employ the eISSN 1533-385X to initiate your request. See also AIAA Rights and Permissions www.aiaa.org/ randp.

*Professor, Laboratory of Vibration and Acoustics, EA677; jerome. antoni@insa-lyon.fr.

${ }^{\dagger}$ Assistant Professor, Laboratory of Vibration and Acoustics, EA677.

*Research Engineer, Laboratory of Vibration and Acoustics, EA677.

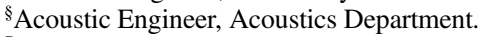

${ }^{\top}$ Acoustic Engineer.

**Deputy Director.

${ }^{\dagger}$ Director.

$\begin{array}{lll}S_{r r^{\prime}}(\alpha, f) & = & \text { order-frequency spectral cross correlation between } \\ & & \text { signals } r(t) \text { and } r^{\prime}(t) \\ t & = & \text { time, } \mathrm{s} \\ \boldsymbol{v}_{k} & = & \text { th eigenvector } \\ w(t) & = & \text { kernel of short-time (angular) Fourier transform } \\ X & = & \text { matrix of short-time Fourier coefficients of signals } \\ & x_{m}(t), \text { where } m \text { is equal to } 1, \ldots, M \\ X_{m i}\left(f_{k}\right) & = & \text { short-time Fourier transform of signal } x(t) \\ X_{m i}\left(f_{k} ; \alpha\right) & = & \text { short-time angular Fourier transform of signal } x(t) \\ x(t) & = & \text { broadband part of aeroacoustic signal } \\ y(t) & = & \text { measured aeroacoustic signal } \\ \alpha & = & \text { cyclic order (counts per revolution) } \\ \gamma & = & \text { spectral bandwidth of kernel } \\ \gamma_{x x}^{2}(\alpha, f) & =\text { order-frequency spectral coherence of signal } x(t) \\ \Delta & = & \text { time shift in short-time (angular) Fourier transform } \\ \Theta & = & \text { angular period, rad } \\ \theta & = & \text { reference angle, rad } \\ \lambda_{k} & = & k \text { th eigenvalue } \\ \tau & = & \text { time lag, s } \\ \chi^{2} & =\text { chi-square distribution }\end{array}$

Subscripts

$i=$ time index in short-time (angular) Fourier transform

$k=$ frequency index in short-time (angular) Fourier transform

$m \quad=\quad$ sensor number

\section{Introduction}

I THE presence of rotating machines (e.g., turbomachines), 1 aeroacoustic measurements often comprise a complex mixture of tonal and broadband components. Separate analysis of these components is of importance for engineers, as they carry different elements of information. Such information is crucial for better understanding the physics of the phenomena, for diagnosis, or for updating numerical models. Extraction of tonal components is the first step toward the separation of sound sources in aeroacoustic signals. 
Because of their marked properties [high spatial coherence and pointwise power spectral density (PSD)], they are the easiest components to be extracted as opposed to broadband components. Several methods have been proposed in the literature to do so, with various degrees of refinement [1-3]. Once removed from the signals, they leave the place to the broadband content of the signals [4-7]. Far fewer works have attempted to further decompose broadband signals into more constituents that originate from distinct physical phenomena or sound sources, at least without explicit knowledge of the propagation mechanisms. (This remark is to exclude a large family of methods, which amount to invert a physical model.) The aim of the present work was to propose a method that, as a complement to the extraction of tones, also enables the decomposition of aeroacoustic signals into nonstationary broadband components attached to rotors and a residual stationary component that contains the background noise. The usefulness of such a decomposition is manifold. It is apt to provide more insight into noise-generating mechanisms to better quantify the contribution of broadband noise attached to specific rotors to the overall noise level or with respect to the tonal part, or it can be used as a preprocessing step to denoise the signals before subsequent analyses, for instance in acoustic imaging. It is noteworthy that the proposed method works at a higher level than phasedarray imaging techniques. It is independent of any wave propagation model and, as such, neither its aim nor its principle is to localize sources in space. (The proposed method is disconnected from beamforming both in the objectives and in the principles. It primarily aims at extracting the contribution of cyclostationary broadband components at the sensors, whereas beamforming aims at localizing sources in space. It is model free, whereas beamforming relies on the inversion of a wave propagation model. It potentially works with only one sensor, whereas beamforming requires an array of microphones.)

The broadband part of aeroacoustic signals has a rich informative content. It typically encompasses unsteady flow, the turbulence produced by bodies moving at high speed, the interaction of these bodies with turbulent flows, the diffraction of waves in turbulent shear layers, etc. [8,9]. The relative contribution of broadband noise as compared to tonal noise actually tends to increase in modern designs of turbomachines $[10,11]$.

Contrary to tones, broadband components are characterized by continuous PSDs. They are modeled as random processes, with finite power but unpredictable (i.e., nondeterministic) trajectories in time. On the one hand, phenomena that globally contribute to background noise are well modeled by stationary stochastic processes (i.e., whose statistics are time invariant). On the other hand, phenomena that are produced by or that interact with rotating parts of the machine are likely to be periodically modulated in phase (Doppler effect) and in amplitude (time-varying intensity). The corresponding stochastic processes are well modeled as cyclostationary (i.e., with periodically varying statistics; see [12] for an early introduction to cyclostationary processes and [13] for a general introduction to some of their applications in vibrations and acoustics). The cyclostationary property of aeroacoustic signals has been recognized in some early references [14-16]; yet, it has rarely been exploited [17, 18].

Just as with tones, it should be reminded that the periodicity of the modulations of broadband noise is with respect to the angle of rotation of the rotor. However, because aeroacoustic phenomena are described by partial differential equations that involve time, the carrier takes time as its descriptive variable. This class of processes has been defined as angle-time cyclostationary (AT-CS) [19]. It asks for some specific treatments, according to two descriptive variables or their dual variables in the Fourier domain, the frequency in hertz (the dual variable of time), and the cyclic order (the dual variable of angle, as a fraction of the reference rotation) in "counts per revolution." Besides, if only second-order statistics are of concern (i.e., autocorrelation function or related spectral quantities) as is the case in this paper, the stochastic processes are said second-order angle-time cyclostationary (AT-CS2). By comparison, tonal components are referred to as first-order angle-time cyclostationary, as their firstorder statistics (i.e., expected value) are angle periodic.

The aim of this paper was to propose a method that can decompose broadband aeroacoustic signals into an AT-CS2 part attached to the rotation of a rotor and a residual stationary part that embodies all other sources and background noise. The method typically applies on the residual signals obtained after removal of the tonal components using a dedicated technique, such as the one in Ref. [1] or in Ref. [3]. As far as the authors know, this topic has rarely been reported in the literature. The aforementioned works $[1,2,4-7]$ have contented themselves with the total broadband part left after removal of tonal components, yet without further decomposing it. A notable exception is Ref. [20], which attempts to fit a model to the periodically timevarying autocorrelation function of a cyclostationary signal produced by a rotating monopole. However, the method is not validated on real experimental data. The approach proposed in the present paper is a continuation of previous works by the authors $[21,22]$. As compared to the latter references, it proposes a generalization, within the framework AT-CS2 processes, that applies to rotating machines in the presence of moderated speed fluctuations. This is of importance from a practical point of view, because such speed fluctuations often exist and are likely to jeopardize cyclostationary-based processing, as will be illustrated in Sec. V. In addition, an important effort has been made to provide a method that is as automated as possible to relieve as much as possible the user of the difficult choice of setting up parameters. Because the method is computationally demanding, the corresponding time saved by the user, as compared to a trialand-error approach, can be substantial. A unique outcome of the proposed method is to make possible the resynthesis of the extracted AT-CS2 component in the time domain. According to the authors' knowledge, there is no such equivalent in the literature.

The principle of the proposed approach is to use the property that AT cyclostationarity produces correlations between order-shifted spectral components that do not exist for stationary processes. By building a regression model based on these correlations, the AT-CS2 components can be predicted to some extent and extracted from stationary noise. When several signals are measured simultaneously, as in the case of an array of microphones, the regression may be augmented by including order-shifted spectral components of other signals as well. There are several issues to be investigated in this approach. A first one relates to the number of order-shifted spectral components to include in the regression model. A second issue arises with the consideration of multiple channels, a configuration that might increase the number of regressors to a point where estimation errors may predominate. As well known in regression analysis, the dimension of the model has to achieve a balance between bias and estimation errors. This is solved by forcing a reduced rank in the model $[23,24]$, which corresponds to the number of virtual sources that can reproduce an equivalent acoustical field.

The paper is organized as follows. Section II introduces the signal model and briefly summarizes the theory of AT-CS 2 signals. It then introduces the short-time angular Fourier transform (STAFT), an order-shifted version of the short-time Fourier transform (STFT), which plays a key role in the proposed method. The principles of the extraction of AT-CS2 broadband noise are discussed in Sec. III. The monochannel case is first investigated for its simplicity before addressing the multichannel case. Section IV strives to make the method as stand-alone as possible by providing rules for automatic settings of the parameters. Section $\underline{V}$ validates the method on synthetic data. Finally, Sec. VI illustrates the extraction of AT-CS2 broadband components on counter-rotating open rotor (CROR) data.

The following notations are used throughout the documents. Bold lowercase letters are used to denote vectors. Bold uppercase letters are used to denote matrices. The imaginary number is noted $j$. In addition, $\|\boldsymbol{a}\|$ stands for the $\ell_{2}$ norm of vector $\boldsymbol{a}$ and $\|\boldsymbol{A}\|$ for the Frobenius norm of matrix $\boldsymbol{A} . \boldsymbol{A}^{H}$ is the Hermitian transpose (i.e., conjugate transpose) of matrix $\boldsymbol{A}$; $\operatorname{trace}\{\boldsymbol{A}\}$ stands for the trace of matrix $\boldsymbol{A} . \mathbb{E}$ is the expectation operator, and $\delta$ stands for Dirac distribution. In general, the symbol ^over a quantity will indicate that this quantity is estimated, for example, $\hat{\theta}$ is an estimate of $\theta$.

\section{Theoretical Background}

This section introduces the notations and the main tools that are used to achieve the extraction of cyclostationary broadband 
components. The signal model related to the broadband part is first revisited and the concept of AT-CS2 is discussed. Next, the orderfrequency spectral correlation (OF-SC) is introduced, as required to calculate the correlation due to angle-periodic modulations, together with the STAFT on which all estimates are rooted.

\section{A. Signal Model and Angle-Time Cyclostationarity}

The signal model is hereafter given in the discrete time setting, in the general case where multiple measurements are simultaneously available (e.g., as returned by an array of microphones). The signal measured by the $m$ th sensor at time instant $t$ is denoted by $x_{m}(t)$. It is composed of the superposition of the signal of interest (SOI), say $r_{m}(t)$, and of the background noise, say $n_{m}(t)$, such that

$$
x_{m}(t)=r_{m}(t)+n_{m}(t), \quad m=1, \ldots, M, \quad t \in \mathbb{R}
$$

[The notation $r_{m}(t)$ is to reflect that the SOI in the paper is assumed purely random.] the SOI and the noise are assumed to be broadband random processes, with continuous but unspecified PSDs. \{The measurements $x_{m}(t)$ are, for instance, the residual signals obtained after subtraction of tonal components, in a preprocessing step, as demonstrated in $[5,13,25]$. $\}$ So far, no assumption is required concerning the probability density functions of the SOI and of the background noise, except some mild conditions for the short-time Fourier coefficients to tend to Gaussianity [23]. The two components $r_{m}(t)$ and $n_{m}(t)$ are, however, characterized by different statistical properties, hereafter denoted as $\mathrm{H} 1$ and $\mathrm{H} 2$, which will make their separation possible:

1) H1: the SOI $r_{m}(t), m=1, \ldots, M$ jointly exhibit second-order angle-time cyclostationarity (AT-CS2), with cyclic orders listed in a nonempty set $\mathcal{A}$ (see next section for a formal definition of the concept of AT-CS2).

2) $\mathrm{H} 2$ : the noise terms $n_{m}(t), m=1, \ldots, M$ are jointly stationary.

Although the aforementioned model allows spatial correlation between sensors for both the SOI and the noise, it is not a necessary condition. However, when true for the SOI, this property will result in better separation of the two components by means of spatial filtering, as explained in Sec. III.

\section{B. Second-Order Angle-Time Cyclostationarity}

Angle-time cyclostationarity, as introduced in assumption $\mathrm{H} 1$, is a specific form of nonstationarity for random processes that undergo angle-periodic modulations, as imposed by the rotating components of a machine. When restricted to second order, AT-CS is formally defined by means of the angle-time (AT) cross-correlation function, $(\mathbb{R}, \mathbb{R}) \rightarrow \mathbb{R}:$

$$
(\theta, \tau) \mapsto R_{r_{m} r_{m^{\prime}}}(\theta, \tau) \doteq \mathbb{E}\left\{r_{m}(t(\theta)+\tau) r_{m^{\prime}}(t(\theta))\right\}
$$

between two processes $r_{m}(t)$ and $r_{m^{\prime}}(t)$. In the preceding definition, $\mathbb{E}$ stands for the expected value, $\theta$ for the angle of rotation (e.g., of the rotor; a continuous variable in radian), $t(\theta)$ for the time instant corresponding to the value of $\theta$, and $\tau$ for a time lag. A one-to-one relation is assumed between $t$ and $\theta$, which is equivalent to assuming that the speed of rotation $\dot{\theta}=d \theta / d t$ does not change its sign. The two processes $r_{m}(t)$ and $r_{m^{\prime}}(t)$ are said jointly AT-CS2 with cyclic order $\alpha=1 / \Theta$ if their AT cross-correlation function is a periodic function of angle $\theta$, that is

$$
R_{r_{m} r_{m^{\prime}}}(\theta, \tau)=R_{r_{m} r_{m^{\prime}}}(\theta+\Theta, \tau)
$$

for some angular period $\Theta$. A typical example of an AT-CS2 signal is provided by random processes whose cross-correlation function has the separable form:

$$
R_{r_{m} r_{m^{\prime}}}(\theta, \tau)=A(\theta) T_{m, m^{\prime}}(\tau)
$$

with $A(\theta)=A(\theta+\Theta)$ an angle-periodic function.

More generally, two processes $r_{m}(t)$ and $r_{m^{\prime}}(t)$ will be said to jointly exhibit AT-CS2 if their cross-correlation function contains an angle-periodic component. A typical example of a process that exhibits AT-CS2 is given by the periodically amplitude-modulated process $r_{m}(t)=\nu_{m}(t) p(t)$, with $p(t(\theta))=p(t(\theta+\Theta))$ a periodic function of $\theta$ and $\nu_{m}(t)$ and $\nu_{m^{\prime}}(t)$, two jointly stationary random processes with cross-correlation function $T_{m, m^{\prime}}(\tau)$. Therefore

$$
\begin{aligned}
R_{r_{m} r_{m^{\prime}}}(\theta, \tau)= & T_{m, m^{\prime}}(\tau) p(t(\theta)+\tau) p(t(\theta)) \\
= & T_{m, m^{\prime}}(\tau) \underbrace{|p(t(\theta))|^{2}}_{A(\theta)}+\tau T_{m, m^{\prime}}(\tau) \dot{p}(\zeta), \\
& \zeta \in[t(\theta) ; t(\theta)+\tau]
\end{aligned}
$$

where the last equality results from Taylor-Lagrange expansion and evidences the presence of the angle-periodic term $A(\theta)=$ $A(\theta+\Theta)$

The PSD of an AT-CS2 random process cannot be distinguished from the PSD of a stationary process, because it averages out any form of nonstationarity. To preserve the evidence of the presence of periodic modulation, the PSD must be generalized, as explained in the next subsection.

\section{Order-Frequency Cross-Spectral Correlation}

A useful spectral tool for analyzing jointly AT-CS2 processes is the order-frequency spectral cross correlation (OF-SCC) [19], defined as the double Fourier transform (a Fourier transform in $\tau$ followed by a Fourier transform in $\theta$ ) of the AT cross-correlation function, $(\mathbb{R}, \mathbb{R}) \rightarrow \mathbb{C}$ :

$$
S_{r_{m} r_{m^{\prime}}}(\alpha, f)=\lim _{\Phi \rightarrow \infty} \frac{1}{\Phi} \int_{\Phi} \int_{\mathbb{R}} R_{r_{m} r_{m^{\prime}}}(\theta, \tau) e^{-j 2 \pi f \tau} e^{-j 2 \pi \alpha \frac{\theta}{\Theta} \mathrm{d} \theta}
$$

where the "carrier frequency" $f$ is dual of time and the "cyclic order" $\alpha$ (also referred to as the "modulation order") is the dual of angle. [In practice, the continuous variables $\tau$ and $\theta$ will be sampled, and the continuous Fourier transforms will be replaced by their discrete versions (see [19]); the continuous form is here momentarily considered to simplify the presentation.] A nonzero value of the OF-SCC at a couple of frequencies $(\alpha, f)$ means that the processes $r_{m}(t)$ and $r_{m^{\prime}}(t)$ jointly exhibit AT-CS 2 in the form of a common random carrier with spectral content at frequency $f$ and modulated by an angleperiodic function with cyclic order $\alpha$. Note that the OF-SCC at cyclic order $\alpha=0$ returns the classical cross spectrum of processes $r_{m}$ and $r_{m^{\prime}}$. When $m=m^{\prime}$, the quantity $S_{r_{m} r_{m}}(\alpha, f)$ will be simply referred to as the OF-SC of process $r_{m}(t)$. Consequently, $S_{r_{m} r_{m}}(0, f)$ reduces to the classical PSD.

In general, the OF-SCC of jointly AT-CS2 processes will be nonzero for a continuum of values of $f$ over a set of discrete values of $\alpha$. For instance, in the case of the cross-correlation function (4)

$$
S_{r_{m} r_{m^{\prime}}}(\alpha, f)=S_{m, m^{\prime}}(f) \sum_{k \in \mathbb{Z}} c_{k} \delta_{\alpha, k}
$$

where $\delta_{\alpha, k}=1$ if $\alpha=k$ and 0 otherwise; $S_{m, m^{\prime}}(f)$ is the discrete Fourier transform of $T_{m, m^{\prime}}(\tau)$, and $\left\{c_{k} ; k \in \mathbb{Z}\right\}$ is the set of Fourier coefficients of $A(\theta)$. The corresponding set of cyclic orders is $\mathcal{A}=\mathbb{Z}$.

The set of nonzero values of the OF-SCC forms the signature of an AT-CS2 component and makes possible its extraction from other, non-AT-CS2 components, in particular from stationary noise. In other words, the exploitation of nonzero values of the OF-SCC at specific cyclic orders in $\mathcal{A}$ will allow the extraction of the SOI $r_{m}(t)$ from the measurement $x_{m}(t)$ in model (1). This process is explained in the next subsections.

\section{Short-Time Angular Fourier Transform}

In practice, the OF-SCC needs to be replaced by an estimator based on finite length measurements. At the same time, the proposed method will require an invertible transform from the time to the frequency domain. The two requirements are achieved by the STFT. Consider the sampled version at time instants $n / F_{s}, n \in \mathbb{Z}$, of a 
signal $x_{m}(t)$, with $F_{s}$ the sampling frequency; the STFT is defined as

$X_{m i}\left(f_{k}\right)=\sum_{n \in \mathbb{Z}} x_{m}\left(n / F_{s}\right) w\left(\gamma\left((n-i \Delta) / F_{s}\right)\right) e^{-j 2 \pi n f_{k} / F_{s}}, j^{2}=-1$

where $w(\gamma t)$ is a smooth, nonnegative, and symmetric kernel with finite support of length $N_{w}$ and spectral bandwidth $\gamma$, and $X_{m i}\left(f_{k}\right)$ is the STFT coefficient calculated at frequency $f_{k}=k F_{s} / N_{w}$, $k=0, \ldots, N_{w}-1$ and time datum $i \Delta / F_{s}, \Delta \in \mathbb{N}^{+}, i=0, \ldots, I-$ 1 with $I=\left\lfloor\left(L-\left(N_{w}-\Delta\right)\right) / \Delta\right\rfloor$. It is noteworthy that the STFT is invertible, at any time instant $t$ possibly different from $n / F_{s}$, according to the formula

$$
x_{m}(t)=\kappa \sum_{i=0}^{I-1} \sum_{k=0}^{N_{w}-1} X_{m i}\left(f_{k}\right) w\left(\gamma\left(t-i \Delta / F_{s}\right)\right) e^{-j 2 \pi t f_{k}}
$$

with $\kappa=\sum_{n \in \mathbb{Z}} w\left(\gamma n / F_{s}\right)$, provided that the stride $\Delta$ is small enough so that $\sum_{i \in \mathbb{Z}} w\left(i \Delta / F_{s}\right)$ sums up to a constant.

Similarly, let us define the STAFT:

$$
\begin{aligned}
X_{m i}\left(f_{k} ; \alpha\right)= & \sum_{n \in \mathbb{Z}} x_{m}\left(n / F_{s}\right) w(\gamma((n \\
& \left.\left.-i \Delta) / F_{s}\right)\right) e^{-j\left(2 \pi n f_{k} / F_{s}+\alpha \theta\left(n / F_{s}\right)\right)} \dot{\theta}\left(n / F_{s}\right)
\end{aligned}
$$

which is a version of the STFT shifted in frequency by some cyclic order $\alpha$. Then, it can be shown that an estimator of the OF-SCC of processes $x_{m}(t)$ and $x_{m^{\prime}}(t)$ is returned by

$$
\hat{S}_{x_{m} x_{m^{\prime}}}(\alpha, f)=\frac{1}{\left\|w_{\gamma}\right\|^{2} I(L) \Phi(L)} \sum_{i=0}^{I(L)-1} X_{m i}\left(f_{k}\right) X_{m^{\prime} i}\left(f_{k} ; \alpha\right)^{*}
$$

where $\left\|w_{\gamma}\right\|^{2}=\sum_{n \in \mathbb{Z}}\left|w\left(\gamma n / F_{s}\right)\right|^{2}, I(L)$ is the number of snapshots given as an explicit function of the signal length $L$ [as indicated after Eq. ( $\underline{8})]$, and $\Phi(L)=\sum_{n=0}^{L-1} \theta\left(n / F_{s}\right)$ is the angular sector covered during the observation of $L$ samples of the signal. One has

$$
\lim _{\gamma \rightarrow 0} \lim _{L \rightarrow \infty} \hat{S}_{x_{m} x_{m^{\prime}}}(\alpha, f)=S_{x_{m} x_{m^{\prime}}}(\alpha, f)
$$

in the mean square sense [19]. It is noteworthy that the signal transformations introduced in this section are synchronized to angle, yet performed in the time domain, thanks to the knowledge of angle $\theta(t)$ as a function of time $t$. This approach has been found extremely convenient for processing AT-CS2 signals, which, by definition, involve dependencies on both the time and the angular variables, as explained in Sec. II.B. As demonstrated in Sec. V, this can make a definite difference in the presence of speed fluctuations.

\section{Methodology for Broadband Noise Extraction}

This section describes the methodology for extracting an AT-CS2 signal from stationary background noise [i.e., $r_{m}(t)$ from $x_{m}(t)$ according to model (1)] under the assumptions discussed in Sec. II. The idea is to predict the STFT of the SOI by building a regression model on the STAFT coefficients introduced in the previous section. This is first explained in the monochannel case $[M=1 \mathrm{in}$ model(1)], and then extended to the multichannel case $(M>1)$.

\section{A. Principle of the Extraction: The Monochannel Case}

Let us denote $\mathcal{A}^{*}=\left\{\alpha_{l}: \alpha \neq 0 ; l=1, \ldots, K\right\}$ the set of nonzero cyclic orders of the SOI $r_{m}(t)$ (e.g., $\mathcal{A}^{*}=\mathcal{A} /\{0\}$ with $\mathcal{A}$ as defined in assumption H1). On the one hand, because the SOI is AT-CS2, it follows from Sec. II.D that $R_{m i}\left(f_{k}\right)$ is correlated with $R_{m i}\left(f_{k} ; \alpha_{l}\right)$, $l=1, \ldots, K$. On the other hand, since $n_{m}(t)$ is stationary, the correlation between $N_{m i}\left(f_{k}\right)$ and $N_{m i}\left(f_{k} ; \alpha_{l}\right)$ is theoretically nil for any $\alpha_{l} \in \mathcal{A}^{*}$. Therefore, a prediction of $R_{m i}\left(f_{k}\right)$ can be obtained from a linear combination of the STAFT coefficients $X_{m i}\left(f_{k} ; \alpha_{l}\right), \alpha_{l} \in \mathcal{A}^{*}$ of the measured signal. This means that there exists a set of frequency gains $C_{l}\left(f_{k}\right)$ such that

$$
R_{m i}\left(f_{k}\right) \simeq \sum_{l=1}^{K} C_{l}\left(f_{k}\right) X_{m i}\left(f_{k} ; \alpha_{l}\right)
$$

The preceding coefficients may be estimated by least squares:

$$
\begin{aligned}
\left\{\hat{C}_{l}\left(f_{k}\right) ; l=1, \ldots, K\right\}= & \operatorname{Arg} \min _{C_{l}\left(f_{k}\right)} \mid X_{m i}\left(f_{k}\right) \\
& -\left.\sum_{l=1}^{K} C_{l}\left(f_{k}\right) X_{m i}\left(f_{k} ; \alpha_{l}\right)\right|^{2}
\end{aligned}
$$

such that, finally, an estimate of the STFT of the SOI is returned by the cyclic regression

$$
\hat{R}_{m i}\left(f_{k}\right)=\sum_{l=1}^{K} \hat{C}_{l}\left(f_{k}\right) X_{m i}\left(f_{k} ; \alpha_{l}\right)
$$

In turn, an estimate of the SOI in the time domain, $\hat{r}_{m i}(t)$, is obtained from the inverse STFT (9) applied to the Fourier coefficients $\hat{R}_{m i}\left(f_{k}\right)$. This explains how the time signals corresponding to ATCS2 components can be ultimately resynthesized. As a by-product, an estimate of the stationary residue is returned by the difference $\hat{n}_{m i}(t)=x_{m i}(t)-\hat{r}_{m i}(t)$.

\section{B. The Multichannel Case}

The principle of the extraction is now generalized to the multiplechannel case. The multichannel case has the capability to account for the spatial coherence between the sensors, and therefore to better extract the SOI when the latter occupies a subspace of low dimension as compared to the number of channels $M$.

\section{Principle}

Let $X \in \mathbb{C}^{M \times I}$ denote the matrix of STFT coefficients for all sensors and all time indices $\left\{\right.$ i.e., $\left.[\boldsymbol{X}]_{m i}=X_{m i}\left(f_{k}\right)\right\}$. Hence, the matrix equivalent of model (1) reads

$$
\boldsymbol{X}=\boldsymbol{R}+\boldsymbol{N}
$$

Similarly, let us introduce $X_{l} \in \mathbb{C}^{M \times I}$, the matrix of STAFT coefficients for all sensors and all time indices, such that $\left[\boldsymbol{X}_{l}\right]_{m i}=X_{m i}\left(f_{k} ; \alpha_{l}\right)$. Therefore, the aim is to find a set of matrix gains $\boldsymbol{C}_{l} \in \mathbb{C}^{M \times M}$ such that

$$
\begin{aligned}
& \boldsymbol{R} \simeq \sum_{l=1}^{K} \boldsymbol{C}_{l} \boldsymbol{X}_{l}=\boldsymbol{C}_{\mathcal{A}}, \quad \text { with } \quad \boldsymbol{C} \doteq\left[\begin{array}{lll}
\boldsymbol{C}_{1} & \cdots & \boldsymbol{C}_{K}
\end{array}\right] \in \mathbb{C}^{M \times M K} \\
& \text { and } \quad \boldsymbol{X}_{\mathcal{A}} \doteq\left[\begin{array}{lll}
\boldsymbol{X}_{1}^{T} & \cdots & \boldsymbol{X}_{K}^{T}
\end{array}\right]^{T} \in \mathbb{C}^{M K \times I}
\end{aligned}
$$

Thus, the product $\boldsymbol{C} \boldsymbol{X}_{\mathcal{A}}$ is expected to return an estimate of $\boldsymbol{R}$. It is noted that the factors $\boldsymbol{C}$ and $\boldsymbol{X}_{\mathcal{A}}$ have no physical meaning when taken independently; in particular, they are not expected to return any information on the localization of the sources, as opposed to beamforming, for instance.

Although the matrix gains $\boldsymbol{C}_{l}$ can be estimated by least squares as in Eq. (14), a major limitation of this approach is to involve a large number, $K M^{2}$, of (complex-valued) unknowns. This is likely to make it impracticable due to the production of large estimation errors. A solution is hereafter introduced based on a reduced-rank model to squeeze the number of unknowns and, therefore, better condition the regression problem. The idea is to search for an optimal linear combination (i.e., which minimizes the mean square prediction error) of the STAFT coefficients $X_{m i}\left(f_{k} ; \alpha_{l}\right)$ of all sensors $m=1, \ldots, M$ and all cyclic order $l=1, \ldots, K$ that provides a small number $S \ll$ $M$ of explanatory variables on which to regress $X_{m i}\left(f_{k}\right)$. These variables are expressed as $\sum_{l=1}^{K} \boldsymbol{b}_{s l}^{H} \boldsymbol{X}_{l}, s=1, \ldots, S$, with $\boldsymbol{b}_{s l} \in$ $\mathbb{C}^{M}$, and may be interpreted as $S$ virtual sources that are apt to 
reproduce an equivalent acoustical field. The estimate of $\boldsymbol{R}$ is then returned by the cyclic regression:

$$
\hat{\boldsymbol{R}}=\boldsymbol{A} \sum_{l=1}^{K} \boldsymbol{B}_{l}^{H} \boldsymbol{X}_{l}
$$

where $\boldsymbol{A} \in \mathbb{C}^{M \times S}$ and $\boldsymbol{B}_{l}=\left[\boldsymbol{b}_{1, l} \ldots \boldsymbol{b}_{S, l}\right] \in \mathbb{C}^{M \times S}$ such that $\boldsymbol{C}_{l}=$ $\boldsymbol{A} \boldsymbol{B}_{l}^{H}$ and $\boldsymbol{C}=\left[\boldsymbol{C}_{1} \cdots \boldsymbol{C}_{K}\right]$ are matrices of rank $S$. The problem now involves $M S(1+K)$ complex-valued unknowns instead of $K M^{2}$.

\section{Identifiability}

This subsection briefly investigates the identifiability of the proposed model to establish the number of degrees of freedom (i.e., of independent unknowns) to infer. The model involves $2 S^{2}$ indeterminate parameters (real and imaginary parts counted independently) since $\boldsymbol{A} \boldsymbol{B}_{l}^{H}=\boldsymbol{A} \boldsymbol{Z} \boldsymbol{Z}^{-1} \boldsymbol{B}_{l}^{H}$ for any invertible matrix $\boldsymbol{Z} \in \mathbb{Z}^{S \times S}$. (Although the product $\boldsymbol{A} \boldsymbol{B}_{k}^{H}$ is unique, this is not the case for the elements of $\boldsymbol{A}$ and $\boldsymbol{B}_{k}$.) Therefore, the model counts only $2 S(M(K+$ $1)-S$ ) degrees of freedom (real and imaginary parts). The indeterminacy is fixed if $2 S^{2}$ different elements in $\boldsymbol{A}$ and/or $\boldsymbol{B}_{l}$ are imposed. The constraint used in the present work is to impose $\boldsymbol{A}^{H} \Gamma^{-1} \boldsymbol{A}=\boldsymbol{I}$ for some $M \times M$ Hermitian positive-definite matrix $\Gamma$.

\section{Estimation}

Following the direction given in the monochannel case, the identification of the multivariate regression model (18) is carried out by least squares. The estimation of the regression coefficients under constraint of reduced rank is by itself a nontrivial task that can be undertaken in several ways [26]. Let $\boldsymbol{B}^{H}=\left[\begin{array}{lll}\boldsymbol{B}_{1}^{H} & \cdots & \boldsymbol{B}_{K}^{H}\end{array}\right] \in \mathbb{C}^{S \times M K}$ and $\boldsymbol{X}_{\mathcal{A}}$ be defined as in Eq. (17). The aim is thus to estimate matrices $\boldsymbol{A}$ and $\boldsymbol{B}$ that minimize the sum of the square differences between the observation $\boldsymbol{X}$ and the prediction $\boldsymbol{A} \boldsymbol{B}^{H} \boldsymbol{X}_{\mathcal{A}}$. For the sake of generality, the sum of the square differences will be calculated with the weighted Frobenius norm $\left\|\boldsymbol{X}-\boldsymbol{A} \boldsymbol{B}^{H} \boldsymbol{X}_{\mathcal{A}}\right\|_{\Gamma}$, where the convention $\|\boldsymbol{X}\|_{\Gamma}^{2} \doteq$ trace $\left\{\boldsymbol{X}^{H} \Gamma^{-1} \boldsymbol{X}\right\}$ is used with some $M \times M$ positive-definite weighting matrix $\Gamma$. To regularize the problem, a penalization is also put on the Frobenius norm of $\boldsymbol{A} \boldsymbol{B}^{H}$ to force the prediction $\hat{\boldsymbol{X}}$ to remain small when the variance of $\boldsymbol{A} \boldsymbol{B}^{H}$ is high. Therefore, the penalized leastsquares estimate of $\boldsymbol{A}$ and $\boldsymbol{B}$ reads

$$
(\hat{\boldsymbol{A}} \hat{\boldsymbol{B}})=\operatorname{Arg} \min _{\substack{\boldsymbol{A} \in \mathbb{C}^{M \times S} \\ \boldsymbol{B} \in \mathbb{C}^{M K \times S} \\ S \leq M}}\left\|\boldsymbol{X}-\boldsymbol{A} \boldsymbol{B}^{H} \boldsymbol{X}_{\mathcal{A}}\right\|_{\Gamma}^{2}+\eta\left\|\boldsymbol{A} \boldsymbol{B}^{H}\right\|_{\Gamma}^{2}
$$

with $\eta \geq 0$ a regularization parameter.

Now, let us introduce the spectral matrices $\boldsymbol{S}_{X X}=\left(\boldsymbol{X} \boldsymbol{X}^{H}\right) / I \in$ $\mathbb{C}^{M \times M}$ and $\boldsymbol{S}_{X X_{\mathcal{A}}}=\left(\boldsymbol{X} \boldsymbol{X}_{\mathcal{A}}^{H}\right) / I \in \mathbb{C}^{M \times M K}$ whose elements are proportional to the cross spectra $\hat{S}_{x_{m} x_{m^{\prime}}}\left(0 ; f_{k}\right)$ and OF-SCC $\hat{S}_{x_{m} x_{m^{\prime}}}\left(\alpha_{l} ; f_{k}\right)$ [see Eq. (11)], respectively, and $\boldsymbol{S}_{X_{\mathcal{A}} X_{\mathcal{A}}}=\left(\boldsymbol{X}_{\mathcal{A}} \boldsymbol{X}_{\mathcal{A}}^{H}\right) / I$ the $M K \times M K$ augmented spectral matrix. The solution to problem (19) subject to constraint $\boldsymbol{A}^{H} \Gamma^{-1} \boldsymbol{A}=\boldsymbol{I}$ is then returned by (see $[\underline{23}, \underline{24}]$ )

$$
\left\{\begin{array}{l}
\boldsymbol{V}_{S}=\left[\boldsymbol{v}_{1} \cdots \boldsymbol{v}_{S}\right] \\
\hat{\boldsymbol{A}}=\Gamma^{1 / 2} \boldsymbol{V}_{S} \\
\hat{\boldsymbol{B}}=\left(\boldsymbol{S}_{X_{\mathcal{A}} X_{\mathcal{A}}}+\eta \boldsymbol{I}\right)^{-1} \boldsymbol{S}_{X X_{\mathcal{A}}}^{H} \Gamma^{-1} \hat{\boldsymbol{A}}
\end{array}\right.
$$

where $\boldsymbol{V}_{S}$ is the modal matrix in the eigenvalue decomposition

$$
\begin{aligned}
& \boldsymbol{\Gamma}^{-1 / 2} \boldsymbol{S}_{X X_{\mathcal{A}}}\left(\boldsymbol{S}_{X_{\mathcal{A}} X_{\mathcal{A}}}+\eta \boldsymbol{I}\right)^{-1} \boldsymbol{S}_{X X_{\mathcal{A}}}^{H} \Gamma^{-1 / 2}=\sum_{s=1}^{M} \lambda_{s} \boldsymbol{v}_{l} \boldsymbol{v}_{l}^{H}, \\
& \lambda_{1} \leq \ldots \leq \lambda_{M}
\end{aligned}
$$

Equation (20) shows that the reduced-rank regressor $\hat{\boldsymbol{C}}=\hat{\boldsymbol{A}} \hat{\boldsymbol{B}}^{H}$ is the cascade of the classical least-squares solution and a projection on a subspace of reduced dimension $S$. It can be shown that the first column of $\boldsymbol{C A}_{\mathcal{A}}$ in Eq. (17), associated to first virtual source, is the linear combination maximally correlated with $\boldsymbol{X}$; then, the second column, associated to second "source," is the next one maximally correlated with $\boldsymbol{X}$, but uncorrelated with the first one, etc. The eigenvalues $\lambda_{s}, s=1, \ldots, S$ may be interpreted as the strengths of the $S$ virtual sources entering in model (18). As a consequence, the $S$ strongest eigenvalues are selected in forming the projector $\hat{\boldsymbol{A}}$.

The prediction error is thus returned by

$$
\begin{aligned}
\| \boldsymbol{X} & -\hat{\boldsymbol{A}} \hat{\boldsymbol{B}}^{H} \boldsymbol{X}_{\mathcal{A}}\left\|_{\Gamma}^{2}+\eta\right\| \hat{\boldsymbol{A}} \hat{\boldsymbol{B}}^{H} \|_{\Gamma}^{2} \\
& =\operatorname{tr}\left\{\Gamma^{-1} \boldsymbol{S}_{X_{\mathcal{A}} X_{\mathcal{A}}}\right\}-\operatorname{tr}\left\{\Gamma^{-1} \boldsymbol{S}_{X X_{\mathcal{A}}}\left(\boldsymbol{S}_{X_{\mathcal{A}} X_{\mathcal{A}}}+\eta \boldsymbol{I}\right)^{-1} \boldsymbol{S}_{X X_{\mathcal{A}}}^{H}\right\}+\sum_{s=S+1}^{M} \lambda_{s}
\end{aligned}
$$

which clearly shows that the closer the fraction $\sum_{s=1}^{S} \lambda_{s} / \sum_{s=1}^{M} \lambda_{s}$ (the relative strength of the $S$ first virtual sources) is to unity, the better is the estimation of the SOI.

\section{Toward Automated Extraction}

The proposed methodology involves several transformations, each with its parameters. Properly setting these parameters is essential for successfully extracting the AT-CS2 components. This section addresses this issue. It makes the difference between those parameters that can be safely fixed beforehand according to general guidelines and those parameters that are highly data dependent. In the latter case, data-based criteria are proposed for automatically setting the value of the parameters.

\section{A. General Guidelines for Parameter Settings}

All the parameters involved in the algorithms of Sec. III are now listed and discussed.

1) The spectral bandwidth $\gamma$ : while the window shape and the fraction of overlap in the STFT and STAFT can be safely set up a priori, the spectral bandwidth $\gamma$ is a crucial parameter that controls the desired frequency resolution of the analysis. It is obviously data dependent and care should be taken to take it small enough so as to capture the smallest spectral details. At the same time, it is the main lever to reduce the overall computational cost of the algorithms, because a broad bandwidth will limit the number $N_{w}$ of frequencies to be scanned. The recommendation is therefore to set $\gamma$ as large as possible without sacrificing too much the frequency resolution. This compromise can surely be found by visual inspection of the signal spectra. It is noteworthy that $\gamma$ controls the resolution of the carrier frequency $f$ independently of the resolution of the cyclic order $\alpha$, the latter being typically set much finer than the former. Therefore, the setting of the spectral bandwidth does not have to account for the time characteristics of the modulations imposed on the signals.

2) The weighting matrix $\Gamma$ : from a practical point of view, the simplest choice is here to take $\Gamma=\boldsymbol{I}$. Nevertheless, from a theoretical point of view, the optimal weighting matrix is $\Gamma=S_{X X}$ because it minimizes the prediction error. In practice, the feasibility of the latter choice will strongly depend on the conditioning of the spectral matrix $\boldsymbol{S}_{X X}$. A safe compromise might be to take $\Gamma=\operatorname{Diag}\left\{\boldsymbol{S}_{X X}\right\}$ (i.e., a diagonal matrix composed of the diagonal elements of $\boldsymbol{S}_{X X}$ ). It is equivalent to systematically prewhitening all signals before processing.

3) The set of cyclic orders $\mathcal{A}^{*}$ : the set $\mathcal{A}^{*}$ of cyclic orders is used to construct the explanatory variables in the regression model. In theory, the larger the set is, the more accurate is the prediction. Nevertheless, a large set of regressors also accumulates estimation errors and is therefore detrimental to precision. Selecting the right number of cyclic orders to include in $\mathcal{A}^{*}$ is therefore a bias-variance tradeoff. A simple approach is to identify the cyclic orders with the most prominent AT-CS2 signature, as identified by visual inspection of the square magnitude of the (estimated) order-frequency spectral coherence (OF-SCoh) of each measurement $x_{m}(t)$, that is 


$$
\hat{\gamma}_{x_{m} x_{m}}^{2}\left(\alpha_{l}, f\right)=\frac{\left|\sum_{i=0}^{I-1} X_{m i}\left(f_{k}\right) X_{m i}\left(f_{k} ; \alpha_{l}\right)^{*}\right|^{2}}{\sum_{i=0}^{I-1}\left|X_{m i}\left(f_{k}\right)\right|^{2} \sum_{i=0}^{I-1}\left|X_{m i}\left(f_{k} ; \alpha_{l}\right)\right|^{2}}
$$

The OF-SCoh is a normalized version of the OF-SC introduced in Sec. II.C, with values in the range $[0 ; 1]$ : a zero value indicates that the cyclic order $\alpha$ does not contribute to explain $X_{m i}\left(f_{k}\right)$, whereas a unitary value means that the explanatory $X_{m i}\left(f_{k} ; \alpha_{l}\right)$ is perfectly correlated with $X_{m i}\left(f_{k}\right)$, and thus provides a perfect prediction of the latter. In practice, the $(1-p)$ level of significance

$$
\gamma_{1-p}^{2}=1-e^{-\frac{x_{2, p}^{2}}{2 l_{\text {eff }}}}
$$

returns an approximated threshold below which values of the OF-SCoh are deemed statistically nil (see the Appendix). In the preceding equation, $\chi_{2, p}^{2}$ is the $p$ quantile of the chi-square with two degrees of freedom, and $I_{\text {eff }}$ is the effective number of snapshots (as given in the Appendix).

If there is strong presumption that the explanatory variables $X_{m i}\left(f_{k} ; \alpha_{l}\right)$ are themselves correlated for different values of $\alpha_{l} \in \mathcal{A}^{*}$, the OF-SCoh may be advantageously replaced by its "partial" versions.

Another strategy is to rely on an automated statistical test that incrementally includes a new cyclic order until it does no longer reduce the prediction error. This test is described in Sec. IV.B.1.

4) The number of AT-CS2 sources $S$ : in theory, this parameter can be inferred from the number of dominant eigenvalues $\lambda_{l}$ in Eq. (21). However, in practice, it is often observed that the eigenvalues have a gradually decreasing spectrum without clear distinction of the "signal" zone. The selection of $S$ places a similar bias-variance tradeoff as the selection of $\mathcal{A}^{*}$. An automated statistical test is discussed in Sec. IV.B.2.

\section{B. Automatic Settings}

As discussed previously, the selection of the number of cyclic orders and of AT-CS2 sources is an important issue that conditions the success of the proposed algorithms. To alleviate the user's responsibility in this difficult choice, two statistical tests are introduced that intend to automate this task. They are both based on an incremental inclusion of cyclic orders and AT-CS2 sources as long as they significantly reduce the prediction error.

\section{Automatic Selection of Cyclic Orders}

Let us first rank the candidate cyclic orders in a set $\mathcal{A}^{\prime}$ from the a priori most significant one to the least significant one. (For instance, the cyclic orders $\alpha_{k}=k$ may be ranked according to the harmonic number, i.e., $\mathcal{A}^{\prime}=\left\{\alpha_{1}, \alpha_{-1}, \alpha_{2}, \alpha_{-2}, \ldots, \alpha_{K^{\prime}}, \alpha_{-K^{\prime}}\right\}$.) Next, suppose that a model based on $K\left(1 \leq K<K^{\prime}\right)$ cyclic orders has been accepted, denoted as $\mathcal{M}_{K}=\left(\left\{\boldsymbol{C}_{l} ; l=1, \ldots, K\right\}\right)$. The test consists in checking if the inclusion of an $(K+1)$ th order will significantly reduce the prediction error of the model. If it does not, then $K$ cyclic orders are retained, which eventually constitute set $\mathcal{A}^{*}$. If it does, then model $\mathcal{M}_{K+1}=\left(\mathcal{M}_{K} \bigcup\left\{\boldsymbol{C}_{K+1}\right\}\right)$ is accepted and tested for the inclusion of an $(K+2)$ th cyclic order. Hence, each iteration amounts to testing the hypothesis:

$$
H_{0}: C_{K+1}=0 \text { in } \mathcal{M}_{K+1}
$$

It is proved in the Appendix that hypothesis $H_{0}$ is to be rejected, at the level of significance $(1-p)$, if

$$
\ln \left(\frac{\hat{\sigma}_{K}^{2}}{\hat{\sigma}_{K+1}^{2}}\right)>\frac{1}{2 I_{\mathrm{eff}} M} \chi_{\nu, 1-p}^{2}
$$

with

$$
\hat{\sigma}_{K}^{2}=\frac{1}{I_{\text {eff }}}\left\|\boldsymbol{X}-\sum_{l=1}^{K} \boldsymbol{C}_{l} \boldsymbol{X}_{l}\right\|^{2}
$$

and $\chi_{\nu, 1-p}^{2}$ the $(1-p)$ quantile of the chi-square with $\nu=2 S M$ degrees of freedom.

\section{Automatic Selection of AT-CS2 Sources}

The selection of the number of AT-CS2 sources can be selected in exactly the same way as the number of cyclic orders, with $\hat{\sigma}_{k}^{2}$ (with index $k$ referring to the number of cyclic orders) then replaced by $\hat{\sigma}_{S}^{2}$ (with index $S$ referring to the number of sources) in Eq. (26). The number of degrees of freedom to be used in the chi-square quantile on the right-hand side of Eq. $(26)$ is then $\nu=2(M(K+1)-S-1)$.

\section{Numerical Experiments}

This section aims at validating the proposed methodology by means of numerical experiments. An AT-CS2 signal is synthesized by simulating the radiation of a rotating monopole to an array of microphones. The signal length is $10^{5}$ samples, and the sampling frequency is $F_{s}=20 \mathrm{kHz}$. The radius of gyration is $r_{0}=0.25 \mathrm{~m}$, and the distance of the center of rotation to the center of the array is 1 $\mathrm{m}$. The speed of sound is $c_{0}=340 \mathrm{~m} / \mathrm{s}$, and the angular speed of rotation is defined as $\dot{\theta}(t)=2 \pi f_{0}+\varepsilon(t)$, where $f_{0}=90 \mathrm{~Hz}$ is the mean frequency and $\varepsilon(t)$ is a first-order autoregressive process, $\varepsilon(t)=0.999 \varepsilon\left(t-1 / F_{s}\right)+\nu(t)$, driven by stationary white Gaussian noise $\nu(t)$. The standard deviation of $\dot{\theta}(t)$ is about one per mil, which is enough to invalidate methods that do not explicitly consider angle rather than time as the descriptive variable, even though the method proposed in this paper could handle much larger speed fluctuations. The monopole strength is driven by a stationary stochastic process $q(t)=0.8 q\left(t-1 / F_{s}\right)+\varepsilon(t)$, where $\varepsilon(t)$ is a stationary white Gaussian noise independent of $\nu(t)$. Therefore $q(t)$ is a colored signal with a steadily decreasing PSD. The pressure signal received at a sensor $m$, a distance $d_{m}$ from the center of rotation, is then expressed as

$$
r_{m}(t)=\frac{q\left(t_{m}^{*}\right)}{4 \pi d_{m}\left(\rho_{m}\left(t_{m}^{*}\right)-\left(r_{0} / c_{0}\right) \dot{\theta}\left(t_{m}^{*}\right) \sin \theta\left(t_{m}^{*}\right)\right)}=\frac{q\left(t_{m}^{*}\right)}{4 \pi d_{m}} p_{m}\left(t_{m}^{*}\right)
$$

with $d_{m} \rho\left(t_{m}^{*}\right)=d_{m} \sqrt{\left(1+\left(r_{0} / d_{m}\right) \cos \theta\left(t_{m}^{*}\right)\right)^{2}+\left(\left(r_{0} / d_{m}\right) \sin \theta\left(t_{m}^{*}\right)\right)^{2}}$ the instantaneous distance between the source and the sensor, and $t_{m}^{*}$ the retarded time such that $t=t_{m}^{*}+$ $\sqrt{d_{m}^{2}+r_{0}^{2}+2 d_{m} r_{0} \cos \theta\left(t_{m}^{*}\right)} / c_{0}$. As reflected by Eq. (28), the rotation of the source produces strong amplitude modulation at the receiver, owing to the product with the angle-periodic function $p_{m}\left(t_{m}^{*}\right)$, and frequency modulation (Doppler effect) owing to the angle-periodic variations of the retarded time $t_{m}^{*}$, with an angular period of $2 \pi$ radians. This corresponds to a mean period of $11 \mathrm{~ms}$, yet with slight fluctuations in time due to the nonconstant speed of rotation. With the aforementioned settings, the observed modulation depth, defined as $\left(A_{\max }-A_{\min }\right) /\left(A_{\max }+A_{\min }\right)$ with $A_{\max }$ and $A_{\min }$ the maximum and minimum values of the modulation function $r_{m}\left(t_{m}^{*}\right)$ of the signal, is close to $50 \%$. Because the source signal is random, the resulting pressure signal $r_{m}(t)$ is AT-CS2. Its cyclic orders are $\alpha_{k}=k, k \in \mathbb{Z}$, and the number of AT-CS2 sources is $S=1$ (here, the number of independent point sources). Following model (1), the SOI is finally obscured with stationary white Gaussian noise $n_{m}(t)$ according to a specified signal-to-noise ratio (SNR), leading to the "measured" signal $x_{m}(t)=r_{m}(t)+n_{m}(t)$.

Figure 1a displays the square magnitude of the OF-SCoh, $\hat{\gamma}_{x_{1} x_{1}}^{2}\left(\alpha_{k}, f\right)$, as defined in Eq. (23), computed at the center of the array (say $m=1$ ) for $\alpha_{k}=k, k=1, \ldots, 10$ (note the symmetry $\left.\hat{\gamma}_{x_{1} x_{1}}^{2}\left(\alpha_{k}, f\right)=\hat{\gamma}_{x_{1} x_{1}}^{2}\left(-\alpha_{k}, f\right)\right)$, under an SNR of $0 \mathrm{~dB}$. The OF-SCoh is well able to evidence the presence of AT-CS2 even in this high level of noise; there are at least five cyclic orders that protrude in a large frequency range above the $95 \%$ level of significance computed according to formula (24). By means of comparison, Fig. 1b displays the same OF-SCoh under the assumption of a constant speed of rotation [i.e., when the STAFT entering in Eq. $\underline{(23)}$ is computed according to Eq. (10) with $\theta(t)$ (respectively, $\dot{\theta}(t)$ ) replaced by 


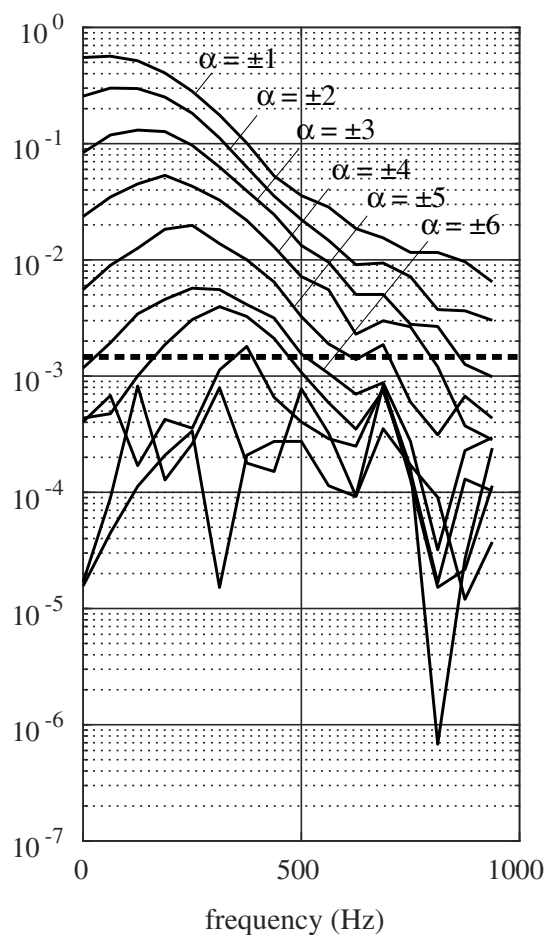

a)

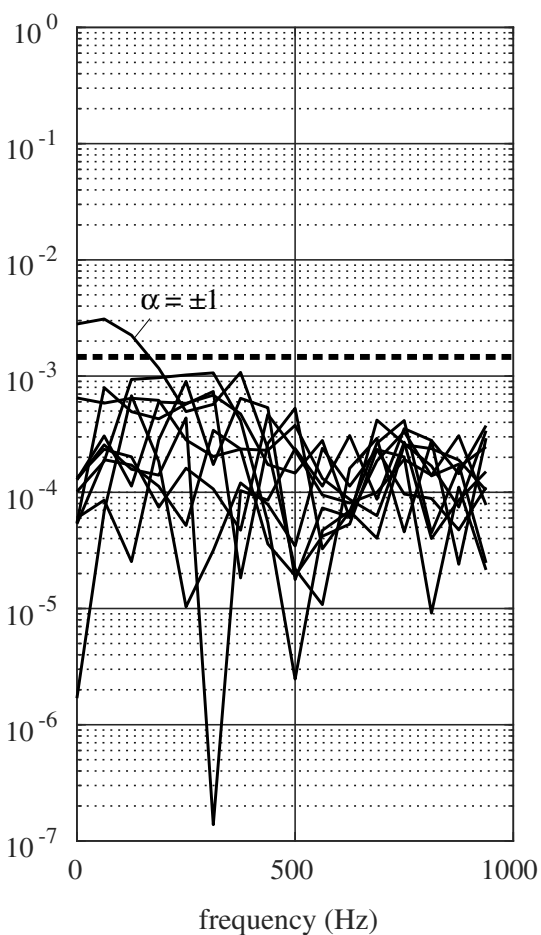

b)

Fig. 1 Square magnitude of the OF-SCoh computed at the center of the array for $\alpha_{k}=k, k=1, \ldots, 10$ together with the $95 \%$ statistical threshold (dotted horizontal line) for $\mathrm{SNR}=0 \mathrm{~dB}$ : a) accounting for the presence of speed fluctuations; b) assuming constant rotation speed $(\Delta f=94 \mathrm{~Hz}$, $\Delta \alpha=0.02 \mathrm{~Hz})$.

$2 \pi f_{0} t$ (respectively, $2 \pi f_{0}$ )]. It is seen that almost all values of the coherence fall below the statistical threshold, a situation that would clearly jeopardize the subsequent use of cyclic regression for extracting the AT-CS2 components, including the methods proposed by the authors in Refs. [21,22]. This illustrates well the necessity to properly account for even small speed fluctuations in the present context.

The first experiment investigates the capability of the proposed method to reconstruct the AT-CS2 component in the absence of additive noise (infinite SNR). The configuration is with $M=20$ microphones randomly positioned in a hemisphere of radius $1 \mathrm{~m}$ from the center of the array and at least $1 \mathrm{~m}$ away from the center of rotation of the source. Based on the former analysis of the OF-SCoh, the set $\mathcal{A}^{*}=\{ \pm 1, \pm 2, \ldots \pm 5\}$ is selected for building the cyclic regression model of Sec. III.B. In the first step, the modulation function $r_{m}\left(t_{m}^{*}\right)$ of Eq. (28) is clipped before multiplication with $q\left(t_{m}^{*}\right)$ so as to impose a maximum modulation depth of $100 \%$ (i.e., $A_{\text {min }}=0$ ). As shown in Fig. 2a, a perfect reconstruction of the SOI is obtained, with a residual stationary part that is virtually nil (see Fig. 2c). This result was found independent of the number of sensors. In the second step, the same experiment is repeated without clipping the modulation function. (As mentioned previously, this corresponds to a modulation depth of about $50 \%$.) It must be understood that part of this signal can now be interpreted as stationary, even though it is produced by the rotating source. This must be so because there exists a continuous transition from an AT-CS2 signal with $100 \%$ modulation depth to a stationary signal, that is, an AT-CS2 signal with $0 \%$ modulation depth. Such a transition is, for instance, observed when decreasing the radius of gyration $r_{0}$ toward zero in Eq. (28). Therefore, in the presence of a moderate modulation depth, part of the source contribution will not be extracted, but left in the stationary residual part. This is illustrated in Fig. $2 b$, where the reconstructed AT-CS2 signal truly corresponds to the modulated part, but slightly differs from the actual signal; as expected, the difference shown in Fig. $2 \mathrm{~d}$ is purely stationary and accounts for about $50 \%$ of the total signal. It is emphasized that this difference does not result from a default of the proposed method, but rather indicates how to interpret the broadband AT-CS2 components that it is able to extract. Indeed, the quantity $r_{m}(t)$ in model (1) should really be interpreted as that part in signal $x_{m}(t)$ that can be unambiguously interpreted as AT-CS2 (i.e., the orange curve in Figs. $2 \mathrm{a}$ and $\underline{2 b})$ and $n_{m}(t)$ as the stationary residual (in Figs. 2c and 2d).

The next experiment investigates the capability of the method to extract AT-CS2 components under an unfavorable SNR of $0 \mathrm{~dB}$ for different values of the number $M$ of microphones and of the number $K$ of cyclic orders used in cyclic regression. The AT-CS2 signal with $100 \%$ modulation depth of Fig. 2a is selected for its ease of interpretation. (In this case, the stationary residual is expected to be nil.) The results are displayed in Fig. 3 by means of the normalized rms error (NRMSE):

$$
\mathrm{NRMSE}=\frac{\left(\sum_{m=1}^{M}\left\|\hat{r}_{m}(t)-r_{m}(t)\right\|^{2}\right)^{1 / 2}}{\left(\sum_{m=1}^{M}\left\|r_{m}(t)\right\|^{2}\right)^{1 / 2}}
$$

where $\hat{r}_{m}(t)$ stands for the estimate of $r_{m}(t)$.

Several remarks are in order. First, the smallest NRMSE that can be reached is now 0.215 . Although this is still acceptable, it clearly demonstrates that the presence of additive noise places a fundamental limit to the recovery. Second, it is seen that the number of sensors $M$ has a strong effect on the extraction capability. Whereas the NRMSE is particularly high when only one microphone is used, it is lowered from 0.80 to 0.38 when the number of microphones is increased to $M=5$. A notable improvement is still observed up to at least $M=25$. Third, the NRMSE decreases with the number of cyclic orders up to a certain point where no more gain is expected. As seen in Fig. 4 and in accordance with the discussion of Sec. IV.A, including too many cyclic orders can actually increase the estimation errors. This illustrates the importance of correctly setting this parameter according to the criteria introduced in Sec. IV. In the present case, the statistical test performed on the OF-SCoh of Fig. 1 already indicated that no more than 10 cyclic orders (five positive values plus their opposite) are required in the cyclic regression model. A similar conclusion was also returned by the criterion of Sec. IV.B.1 (not detailed here, yet to be illustrated in the next section). Fourth, for all runs in the experiment, the number of 


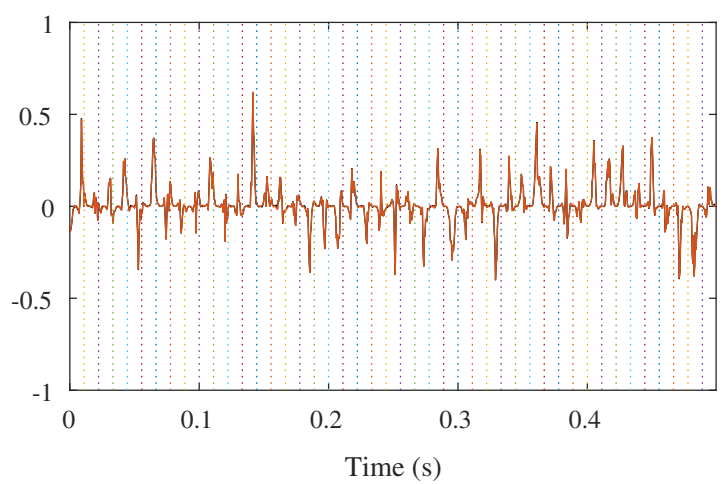

a)

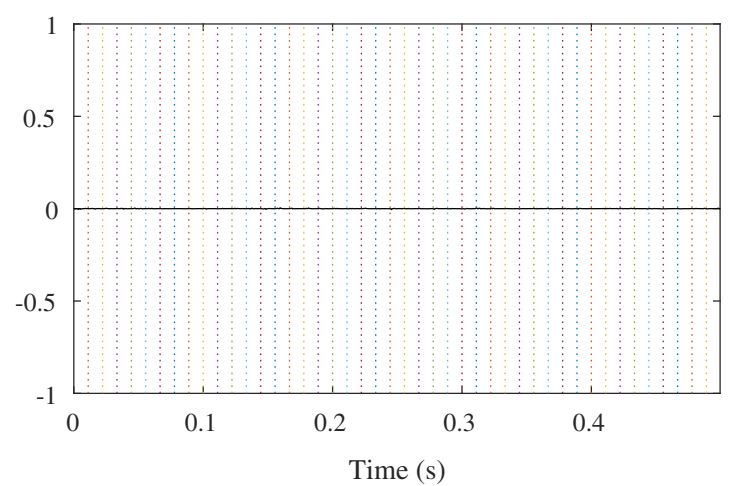

c)

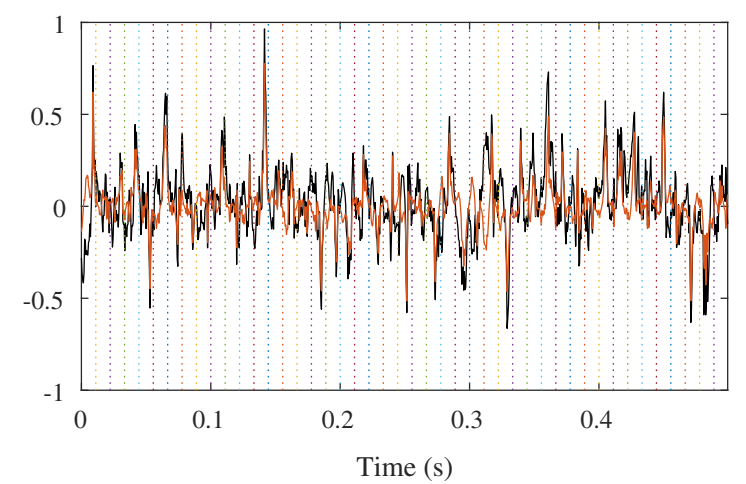

b)

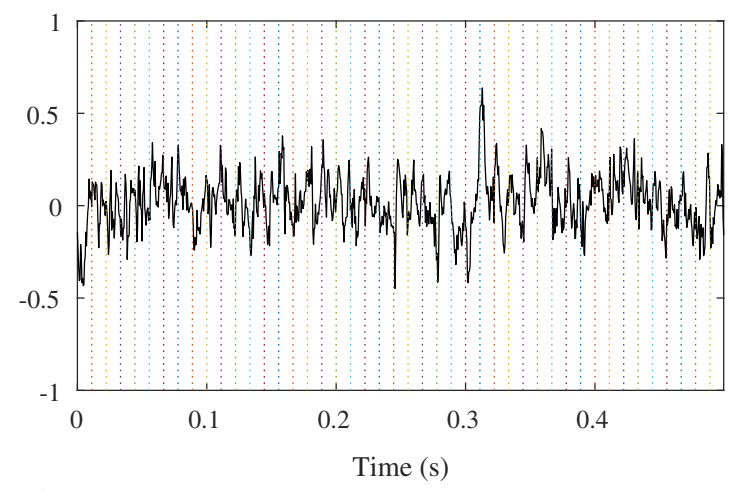

d)

Fig. 2 Actual (black) and reconstructed (orange) signal on the first microphone in the case of a) $100 \%$ modulation depth and b) $50 \%$ modulation depth; c-d) residual stationary parts corresponding to cases a-b); the vertical dotted lines show the succession of periods (infinite SNR, $M=20$ ).

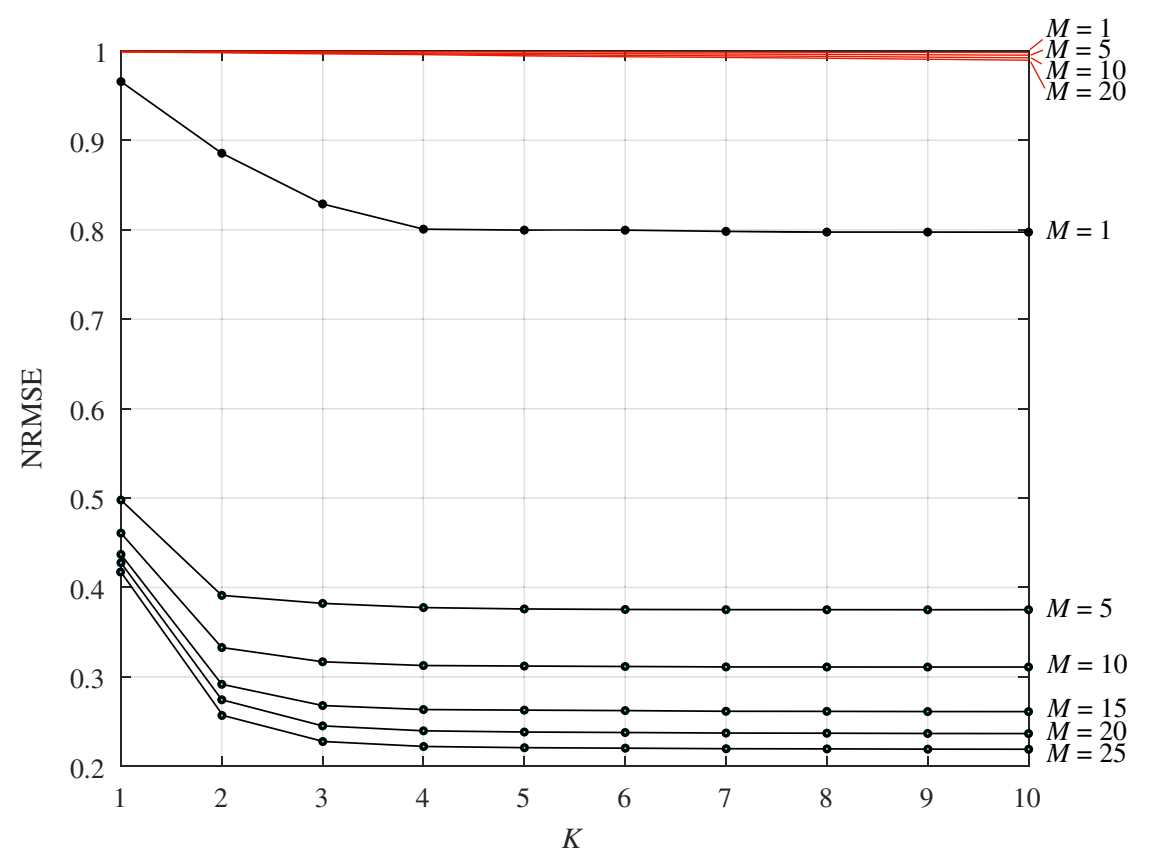

Fig. 3 Normalized rms error as a function of the number $M$ of microphones and of the number $K$ of cyclic orders when extracting an AT-CS2 signal with $100 \%$ modulation depth (black curves); the red curves show the error when the SOI is purely stationary.

AT-CS2 sources was automatically selected according to the criterion of Sec. IV.B.2. The estimate was always 1 or 2 , close to the theoretical value $S=1$. Further experiments not reported here have investigated the configuration with several rotating monopoles driven by independent stationary stochastic processes so as to simulate the case with $S>1$. The results indicated that overestimating the actual number of AT-CS2 sources only slightly increased the NRMSE, while underestimating it led to much larger errors.
Fortunately, the criterion of Sec. IV.B.2 seemed to be conservative in this respect.

The last experiment consists in attempting to extract AT-CS2 components from purely stationary noise [i.e., when the pressure signal $r_{m}(t)$ is set to zero]. As illustrated by the red curves in Fig. 3, the method correctly returns a nil estimate (i.e., an NRMSE nearly equal to one) in this case, even when a large number of microphones are used. 


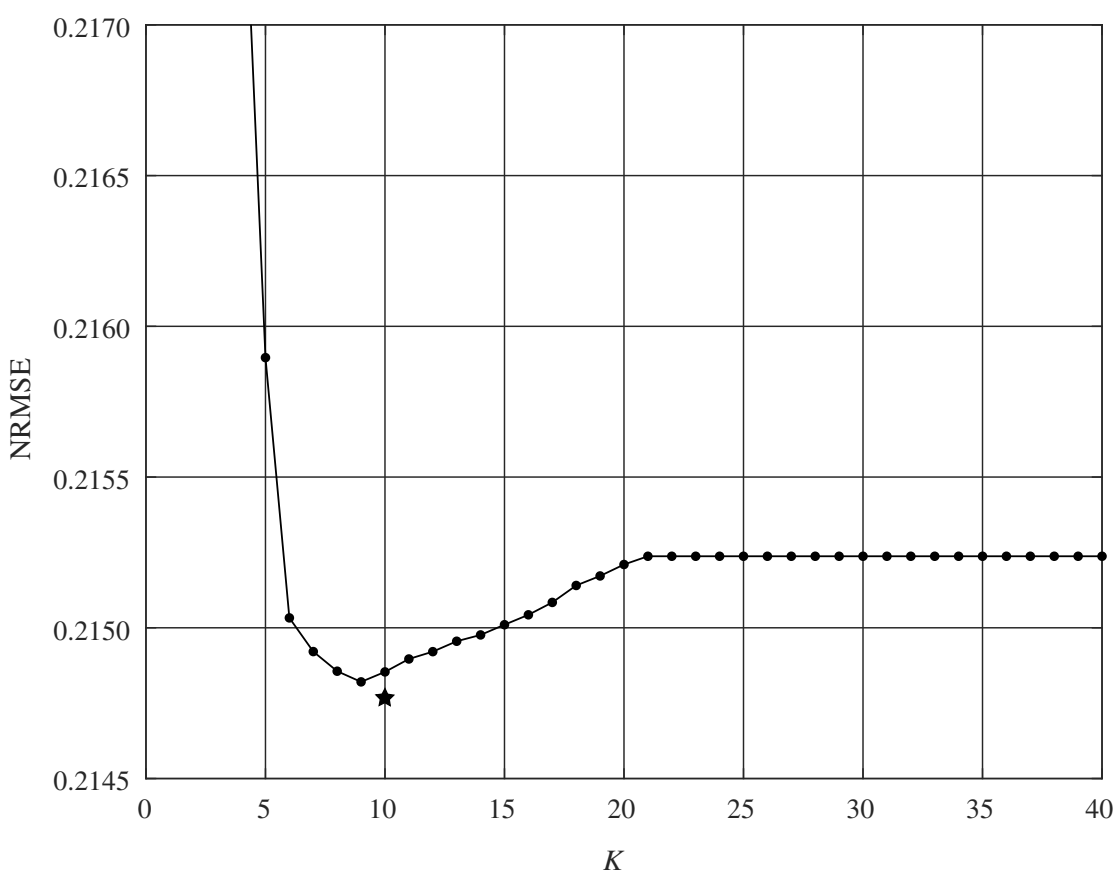

Fig. 4 Zoom of the normalized rms error as a function of the number $K$ of cyclic orders when extracting an AT-CS2 signal with $100 \%$ modulation depth (black curves) and $M=25$ of microphones; the star indicates the optimal number of cyclic orders found by the criteria proposed in Sec. IV.B.1.

\section{Experimental Validation on CROR Data}

The extraction of AT-CS2 components is now illustrated on aeroacoustic signals recorded on an CROR engine in a wind tunnel. The CROR data typically comprise a mixture of tonal and broadband components $[7,11]$. Denoting by $y_{m}(t)$ the signal measured by the $m$ th sensor, by $p_{m}(t)$ the tonal contribution, and by $x_{m}(t)$ the broadband contribution, the signal model reads

$$
\left\{\begin{array}{l}
y_{m}(t)=p_{m}(t)+x_{m}(t) \\
x_{m}(t)=r_{m}(t)+n_{m}(t),
\end{array} \quad m=1, \ldots, M\right.
$$

The tonal contribution $p_{m}(t)$ was extracted in a first step by following the approach described in the companion paper [3] to leave alone the broadband residual $x_{m}(t)$. [The method introduced in Ref. [3] consists in tracking the tones in the angular domain so as to accommodate small speed fluctuations while at the same time recovering to some extent slow random modulations (e.g., due to the effect of turbulence on the propagation of the waves.] The next step is to decompose $x_{m}(t)$ into an AT-CS2 part $r_{m}(t)$ attached to the rotors and a stationary part $n_{m}(t)$ assigned in particular to background noise. It is noted that the AT-CS2 part can be further decomposed into three contributions, say

$$
r_{m}(t)=r_{1, m}(t)+r_{2, m}(t)+r_{12, m}(t)
$$

attached to the rear and front rotors and their interactions, respectively. These components can be theoretically extracted individually, provided that the rotors operate with asynchronous speeds.

\section{A. Presentation of the Data}

The data were collected by a linear array of flush-mounted microphones, placed parallel to the CROR in the wind tunnel (see Figs. 5 and 6). All results are presented hereafter for Mach speed 0.78 and desynchronized rotor speeds, say $f_{1}$ and $f_{2}$, for the rear and front rotors, respectively. The instantaneous angles of rotation of the rear and front rotors were measured by two encoders. In what follows, all frequency axes are normalized by the sampling frequency $F_{s}$.

\section{B. Preliminary Analyses}

The specification of the cyclic regression model (18) requires the list of cyclic orders and the list of neighboring sensors that will act as regressors. Before attempting to answer this question with the help of statistical tests, some physical considerations are first of concern.

Because both the front and rear rotors contribute to the radiated acoustical field, the two families of harmonics $\left\{k \cdot f_{1}, k= \pm 1\right.$, $\pm 2, \ldots\}$ and $\left\{k \cdot f_{2}, k= \pm 1, \pm 2, \ldots\right\}$ must be taken into account in the separation. Because interactions due to modulation of one rotor by the other one exist, it seems at first sight that harmonic combinations of the form $\left\{m \cdot f_{1}+n \cdot f_{2}, m, n= \pm 1, \pm 2, \ldots\right\}$ are also expected. However, it should be kept in mind that cyclic orders are modulation frequencies, and do not include carrier frequencies, and therefore boil down to the same lists $\left\{k \cdot f_{1}, k= \pm 1, \pm 2, \ldots\right\}$ and $\left\{k \cdot f_{2}, k= \pm 1, \pm 2, \ldots\right\}$. This is well confirmed by the inspection of the OF-SCoh (see Fig. 7), where the first harmonics in the families of $f_{1}$ and $f_{2}$ essentially dominate in a wide frequency range.

Taking into account all the harmonics in the families of $f_{1}$ and $f_{2}$ still represents a huge amount of regressors, which is too large to guarantee statistical significance of the separation. Because many of these regressors are likely to be strongly cross correlated, the list can be reduced to only a few of them, which are the most representative. It seems natural to truncate the lists to the first harmonics only (i.e., $\{k$. $\left.f_{1}, k= \pm 1, \pm 2, \ldots, \pm K / 4\right\}$ and $\left\{k \cdot f_{2}, k= \pm 1, \pm 2, \ldots, \pm K / 4\right\}$ with $K$ to be specified). These $K$ potential cyclic orders are to be multiplied by the number of neighboring sensors, say $P$, participating in the regression, thus leading to a regression model with $P K$ regressors of the form:

$$
\hat{R}_{m}(f)=\sum_{k=1}^{K} \sum_{n \in N_{m}} c_{\mathrm{kln}}(f) X_{n}\left(f ; \alpha_{k}\right)
$$

where $\left\{\alpha_{1}, \alpha_{2}, \alpha_{3}, \alpha_{4}, \ldots\right\}=\left\{f_{1},-f_{1}, f_{2},-f_{2}, 2 f_{1},-2 f_{1}, 2 f_{2}\right.$, $\left.-2 f_{2}, \ldots\right\} / F_{s}$, and $N_{m}$ is a set of indices corresponding to $P$ sensors in the neighborhood of sensor $m$. It is important to understand that, according to Sec. III, the regression model (32) intends to extract from measurement $X_{m}(f)$ all spectral components correlated with $X_{n}\left(f ; \alpha_{k}\right)$; when the cyclic orders $\alpha_{k}$ 's pertain to the union set of both rotors, the spectral components that are potentially extracted include not only the direct contributions of the front and rear rotors, but also their interactions. This is surely different from the approach that 


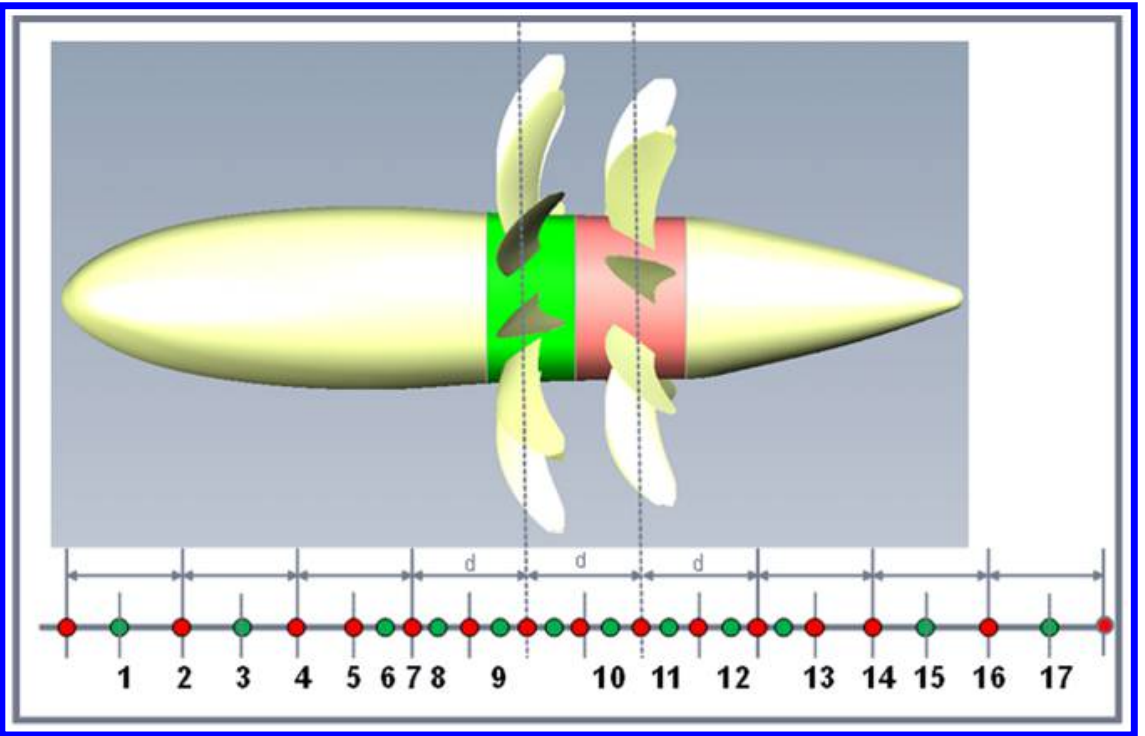

Fig. 5 Schematic of the CROR with the acoustic array; sensors used in the analysis are numbered from 1 to 17.

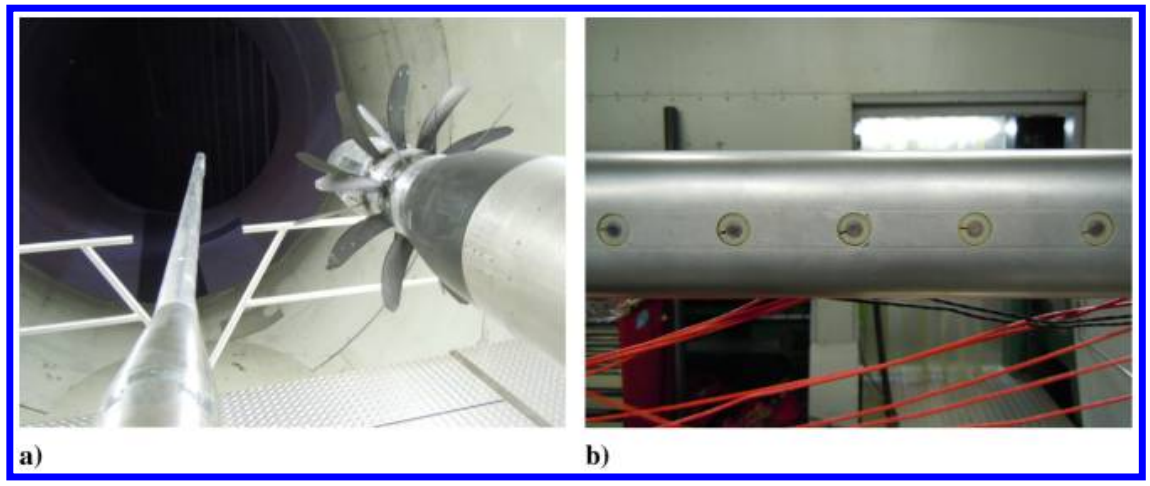

Fig. 6 a) picture of the CROR (rearview) with the acoustic array; b) flush-mounted microphones.

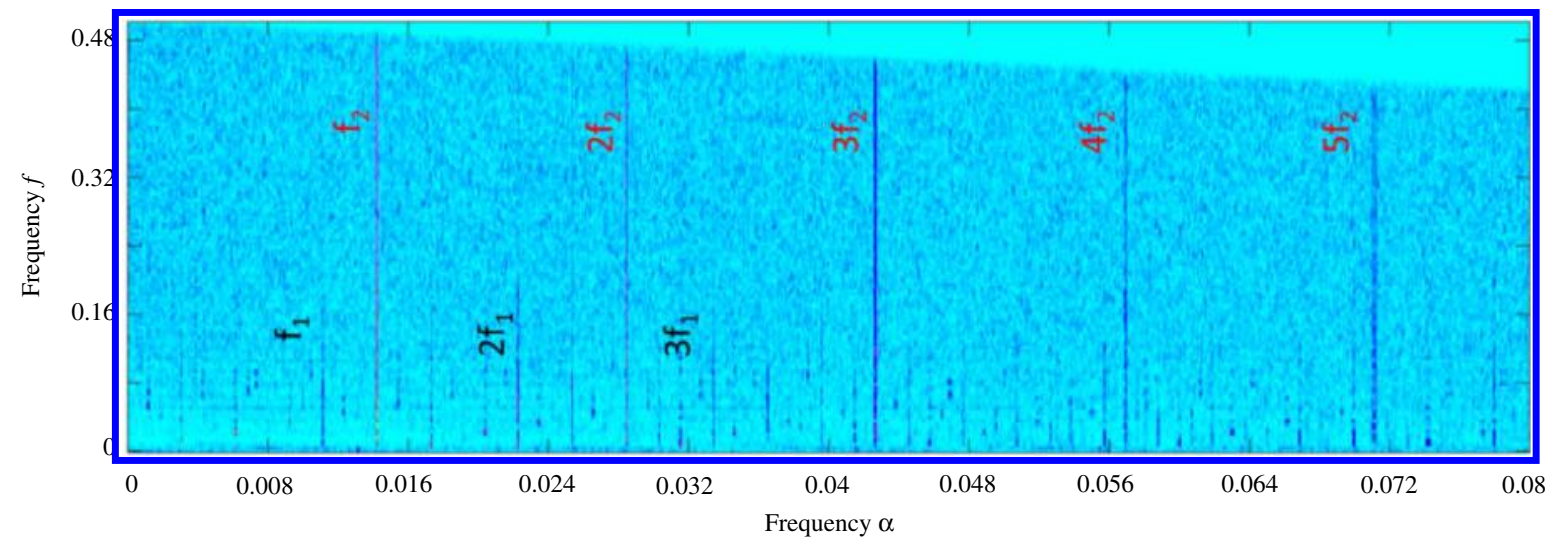

Fig. 7 Order-frequency spectral coherence of sensor $10\left(\Delta f=6 \times 10^{-3} F_{s} ; \Delta \alpha=10^{-5} F_{s}\right)$; distribution over frequency $f$ reveals the carrier spectrum, whereas distribution over $\alpha$ (cyclic order) reveals the modulation spectrum; the color bar grades linearly from 0 (light blue) to 0.1 (dark blue).

would consist in regressing $X_{m}(f)$ independently on the cyclic orders of each rotor, which would not be able to extract all interactions.

Figure 8 displays the coherence between the spectral component $X_{m}(f)$ and the regressors $X_{n}\left(f ; \alpha_{k}\right)$ for $K=8$ and all sensors $\left(N_{m}=\{1, \ldots, M\}\right.$ with $\left.M=17\right)$. It is seen that the coherence is significant only for closely spaced sensors, which reflects the fact that the residual broadband pressure field obtained after subtraction of tones has limited spatial coherence. The spatial correlation length is estimated to be no more than about $3 d$ (with $d$ the spacing between microphones). Moreover, strong coherence exists between cyclic orders within and between groups of spatially close sensors.
These observations are further supported by some statistical tests introduced in Fig. 9 with $N_{m}=\{m\}$ (one and same sensor in the regression model). In Fig. 9a, the multiple coherence function between $X_{m}(f)$ and $\left\{X_{m}\left(f ; \overline{\alpha_{1}}\right), \ldots, X_{m}\left(f ; \alpha_{k}\right)\right\}$ is displayed for increasing values of $k$ and compared to its level of significance at $95 \%$ for sensor 10. (Similar figures have been drawn for the other sensors, but not shown here.) It is seen that the coherence is highly significant below $0.08 F_{s}$. A similar observation holds for the other sensors (not shown), some of them evidencing coherence over a much larger frequency range. Figure $9 \mathrm{~b}$ leads to a similar conclusion with different statistics that measures how the group of regressors 


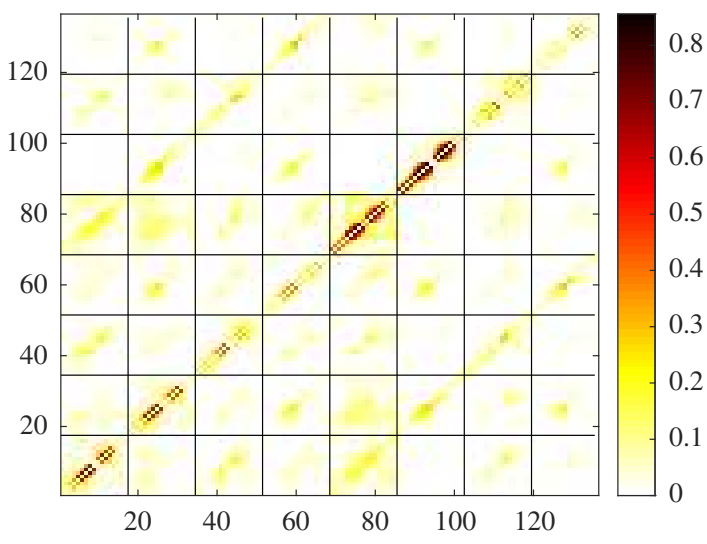

a)

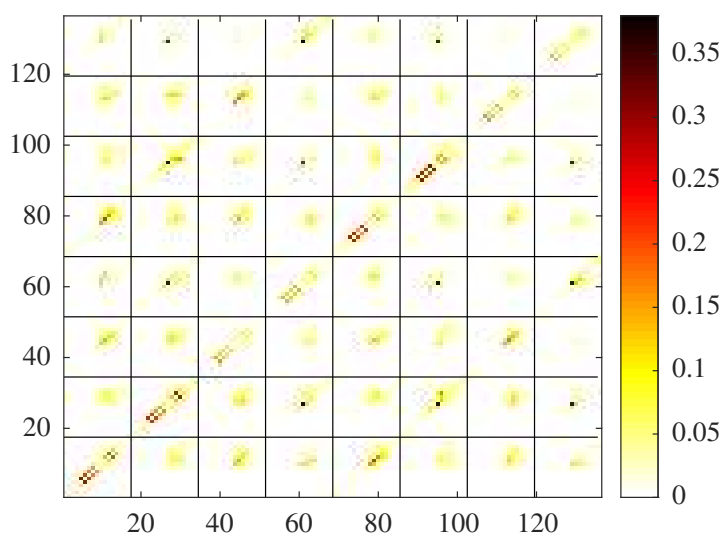

c)

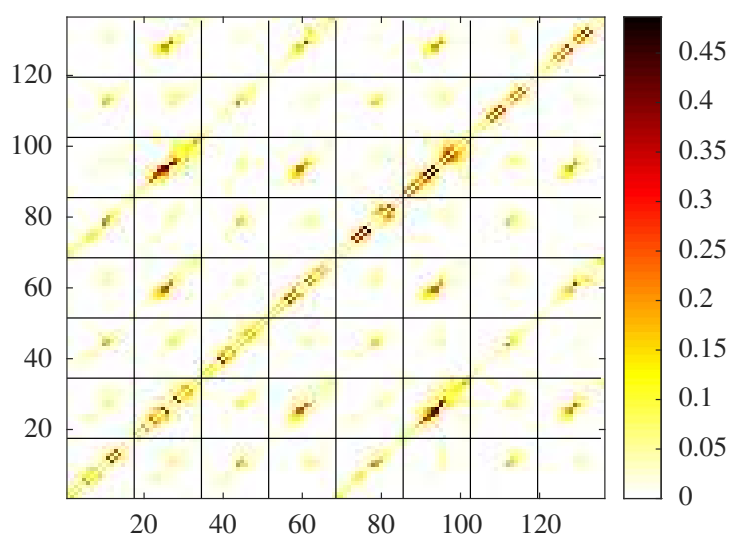

b)

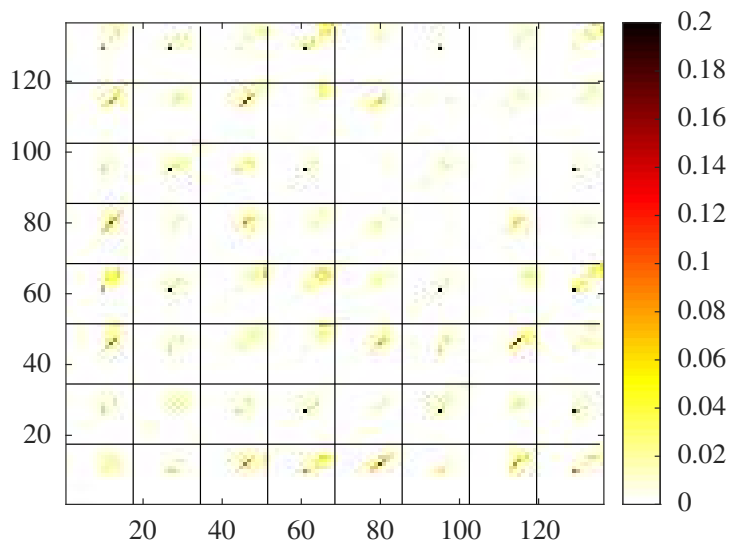

d)

Fig. 8 Coherence between groups of 17 sensors (marked by boxes) at different cyclic orders $\left\{\alpha_{1}, \alpha_{2}, \ldots, \alpha_{8}\right\}=\left\{+f_{1},-f_{1},+f_{2},-f_{2},+\right.$ $\left.2 f_{1},-2 f_{1},+2 f_{2},-2 f_{2}\right\}$; the ordinates of the axes are the values of $17(k-1)+m$, with $m=1, \ldots, 17$ the sensor index and $k=1, \ldots, 8$ the index of the cyclic order $\alpha_{k}$; the main diagonal is removed; carrier frequency is a) $0.035 F_{s}$, b) $0.074 F_{s}$, c) $0.152 F_{s}$, and d) $0.309 F_{s}\left(\Delta f=6 \times 10^{-3} F_{s}\right.$; $\left.\Delta \alpha=10^{-5} F_{s}\right)$.

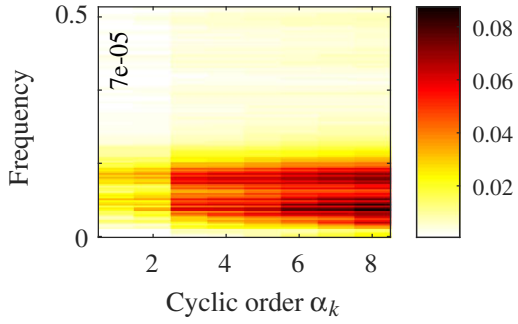

a)

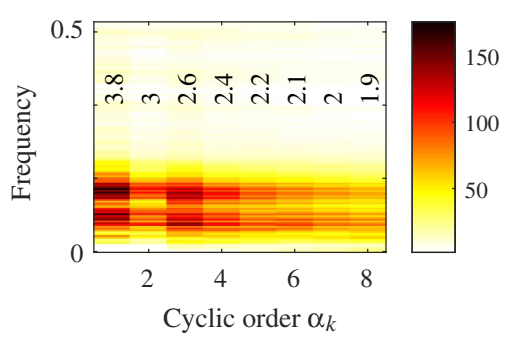

b)

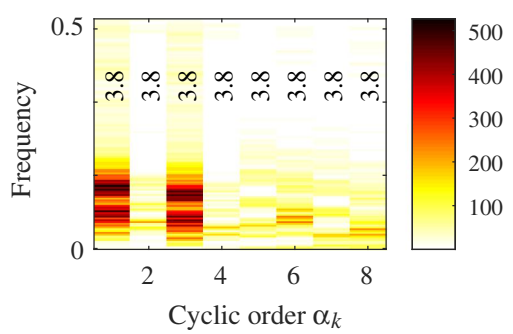

c)

Fig. 9 Analysis of the effect of cyclic orders in the regression model (17) for sensor 10: a) overall coherence function based on the first $k$ cyclic orders in the set $\left\{+f_{1},-f_{1},+f_{2},-f_{2},+2 f_{1},-2 f_{1},+2 f_{2},-2 f_{2}\right\}$; b) statistical contribution of the first $k$ cyclic orders ; c) statistical effect of adding the $k$ th cyclic order to the regression; vertical numbers indicate in each case the levels of significance at $5 \%\left(\Delta f=6 \times 10^{-3} F_{s} ; \Delta \alpha=10^{-5} F_{s}\right)$.

$\left\{X_{m}\left(f ; \alpha_{1}\right), \ldots, X_{m}\left(f ; \alpha_{k}\right)\right\}$ improves the prediction of $X_{m}(f)$, as opposed to using no regressors. Finally, Fig. $9 \mathrm{c}$ shows how the addition of $X_{m}\left(f ; \alpha_{k}\right)$ improves the prediction based on $\left\{X_{m}\left(f ; \alpha_{1}\right), \ldots, X_{m}\left(f ; \alpha_{k-1}\right)\right\}$. Interestingly, harmonics of family $f_{1}$ are more important for sensors 1 and 12 (not shown), whereas harmonics of family $f_{2}$ are more important for sensor 6 (not shown). This illustrates that the relative roles of cyclic orders in the separation are highly dependent on the position of the sensor with respect to the engine.

A last statistical test is provided by the number of significant sources $S$ found in cyclic regression as returned by the model selection criterion of Sec. IV.B.2. The criterion is displayed in Fig. 10 for all the frequency bins in the full frequency range, with $N_{m}=\{1, \ldots, M\}, M=17$, and $K=8$. The criterion regularly decreases with the number of sources and arrives very close to the significance level, even though it does not cross it except for the very first frequencies. Note that, by construction, the number of sources $S$ cannot exceed the number of sensors $M$. This indicates that out of the $P K=136$ regressors, there are at least $S=17$ independent linear combinations of them that can be extracted by cyclic regressions. Yet, because the criterion ends up very close to the significance level, it is likely that much fewer sources could equivalently be used in cyclic regression.

Based on these findings, a second investigation was carried on groups of neighbor sensors only. It was checked that the spatial coherence was well captured within groups consisting of approximately three adjacent sensors. At the same time, reducing the number of sensors in the list of regressors makes possible to increase the number of cyclic orders. A significant correlation was found up to $K=20$, thus leading to a model with $P K=60$ regressors. 


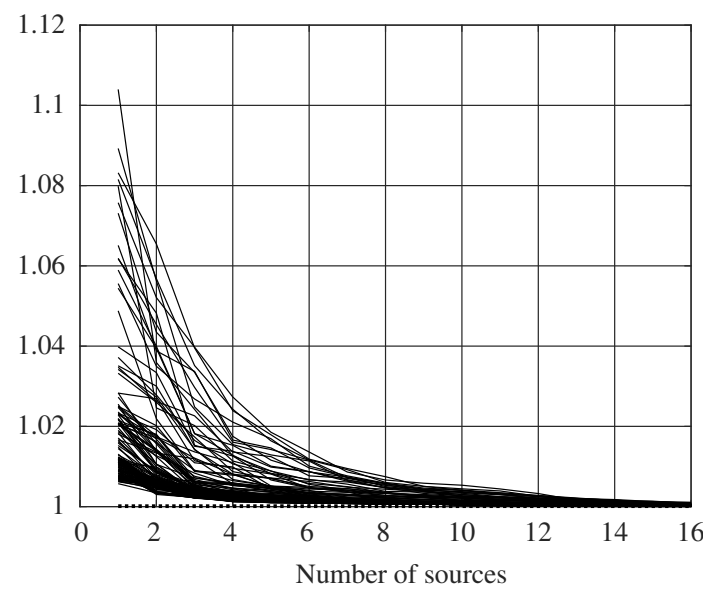

a)

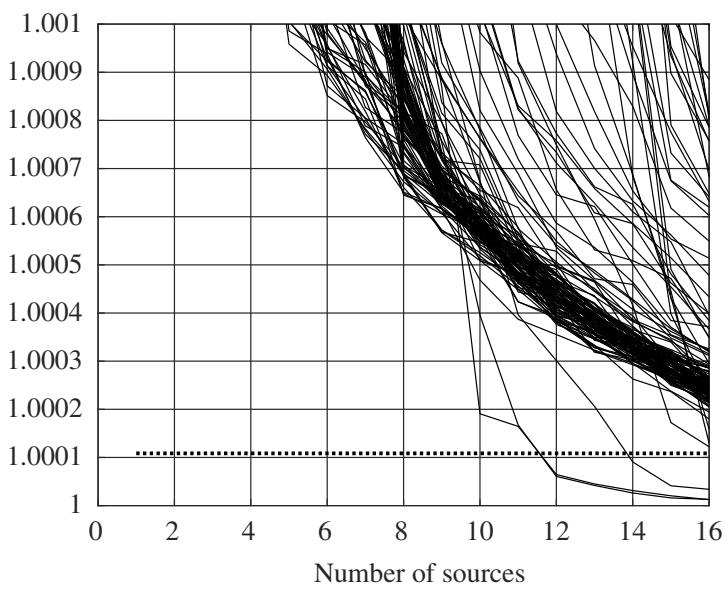

b)

Fig. 10 Ratio of mean square errors with $k$ and $(k+1)$ sources compared to the statistical threshold at risk $5 \%$; right panel $(b)$ is an excerpt of left panel.

\section{Analysis of Separation Results}

The extraction of the AT-CS2 components is now undertaken with the settings found in the previous subsection (i.e., with subgroups of three adjacent sensors with $K=5$ ). A kernel bandwidth of $10^{-3} F_{s}$ is chosen for the STAFT. With these settings, the computation of the spectral matrices, the most demanding task of the method, took approximately $1 \mathrm{~h}$ with an Intel Core i7-4810MQ processor.

The cyclic regression model (32) makes it possible to return an estimation of the total contribution of the AT-CS2 components attached to the front and rear rotors, including their interactions. Once the regression coefficients $c_{\mathrm{kln}}(f)$ have been estimated, it is also possible to use the same regression model to extract only the contribution due to the rear or front rotor. Upon defining the reduced set $K_{1}=\{1,2,5,6,9,10, \ldots\}$ [these indices correspond to the set of cyclic orders $\left\{f_{1},-f_{1}, 2 f_{1},-2 f_{1}, 3 f_{1},-3 f_{1}, \ldots\right\} / F_{s}$ in Eq. (32)]

$$
\hat{R}_{1, m}(f)=\sum_{k \in K_{1}} \sum_{n \in N_{m}} c_{\mathrm{kln}}(f) X_{n}\left(f ; \alpha_{k}\right)
$$

is then an estimate of the acoustical contribution of the rear rotor only. Similarly, with $K_{2}=\{3,4,7,8,11,12, \ldots\}$ [set of cyclic orders $\left\{f_{2},-f_{2}, 2 f_{2},-2 f_{2}, 3 f_{2},-3 f_{2} \ldots\right\} / F_{s}$ in Eq. (32)]

$$
\hat{R}_{2, m}(f)=\sum_{k \in K_{2}} \sum_{n \in N_{m}} c_{\mathrm{kln}}(f) X_{n}\left(f ; \alpha_{k}\right)
$$

is an estimate of the acoustical contribution of the front rotor only. By difference, an estimate of the interactions between the two rotors is returned by

$$
\hat{R}_{12, m}(f)=\hat{R}_{m}(f)-\hat{R}_{1, m}(f)-\hat{R}_{2, m}(f)
$$

Eventually, an estimate of the residual stationary noise is obtained from $\hat{N}_{m}(f)=X_{m}(f)-\hat{R}_{m}(f)$.

By means of an example, Figs. 11 and 12 illustrate the separation results for sensors 10 and 11 , respectively. These are obtained as follows. First, the tonal part (gray line) is extracted by the methodology introduced in Ref. [3]. This leaves a broadband signal (not shown in the figures), comprising AT-CS2 and stationary components, which is then processed with the algorithm introduced in Sec. III. In the first step, the total AT-CS2 part due to both rotors (green line) is extracted on the basis of Eq. (32). Next, this is further decomposed into AT-CS2 part stemming from the front (orange line) and rear rotors (brown dashed line) on the basis of Eqs. (33) and (34). The difference between the total AT-CS2 part and the sum of the individual AT-CS2 parts of each rotor is due to the presence of

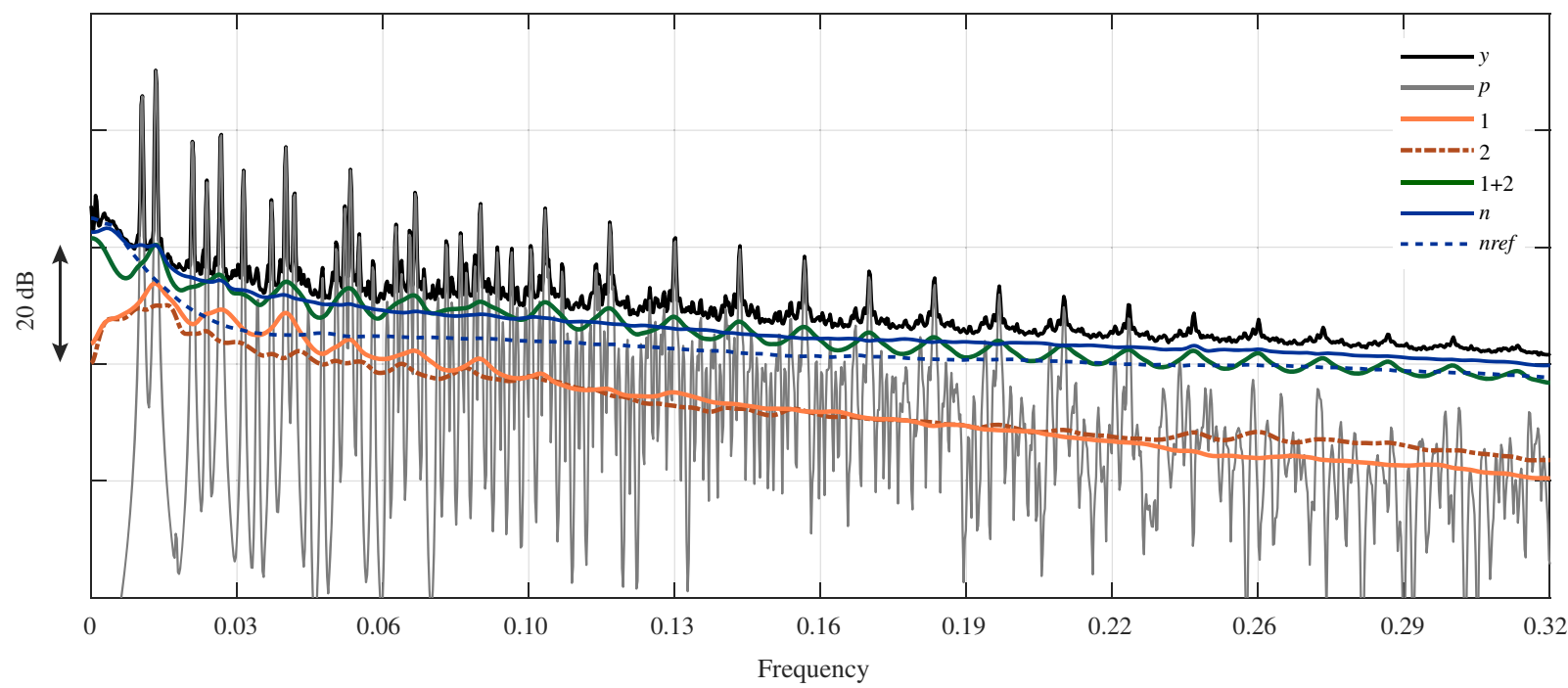

Fig. 11 Power spectral density (arbitrary units) of sensor $10\left(\Delta f=4 \times 10^{-4} F_{s}\right)$; black line, total signal; gray line, extracted tonal part; green line, extracted AT-CS2 part due to front and rear rotors (including interactions); orange line, extracted AT-CS2 part due to rear rotor only; brown dashed line, extracted AT-CS2 part due to front rotor only; blue line, residual stationary noise; blue dotted line, noise reference. 


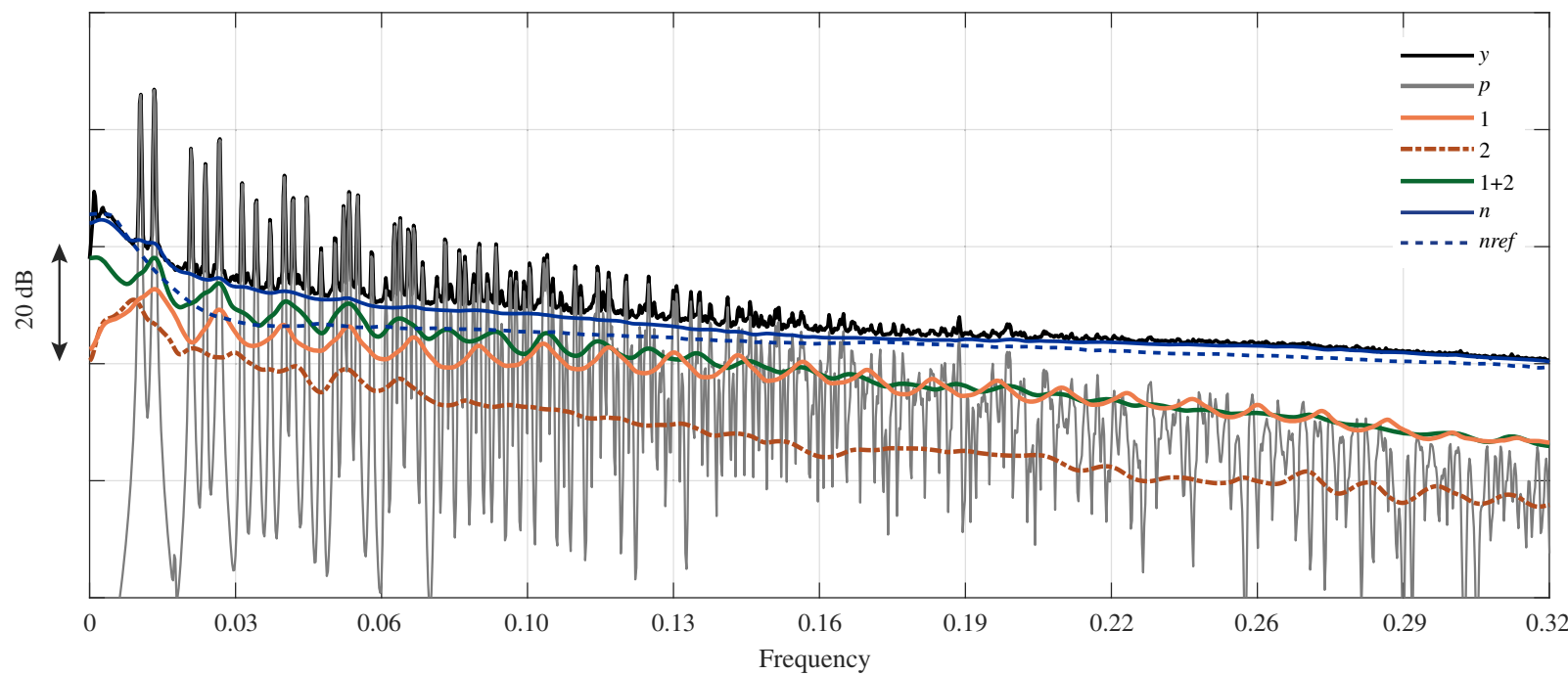

Fig. 12 Power spectral density (arbitrary units) of sensor $11\left(\Delta f=4 \times 10^{-4} F_{s}\right)$; black line, total signal; gray line, extracted tonal part; green line, extracted AT-CS2 part due to front and rear rotors (including interactions); orange line, extracted AT-CS2 part due to rear rotor only; brown dashed line, extracted AT-CS2 part due to front rotor only; blue line, residual stationary noise; blue dotted line, noise reference.
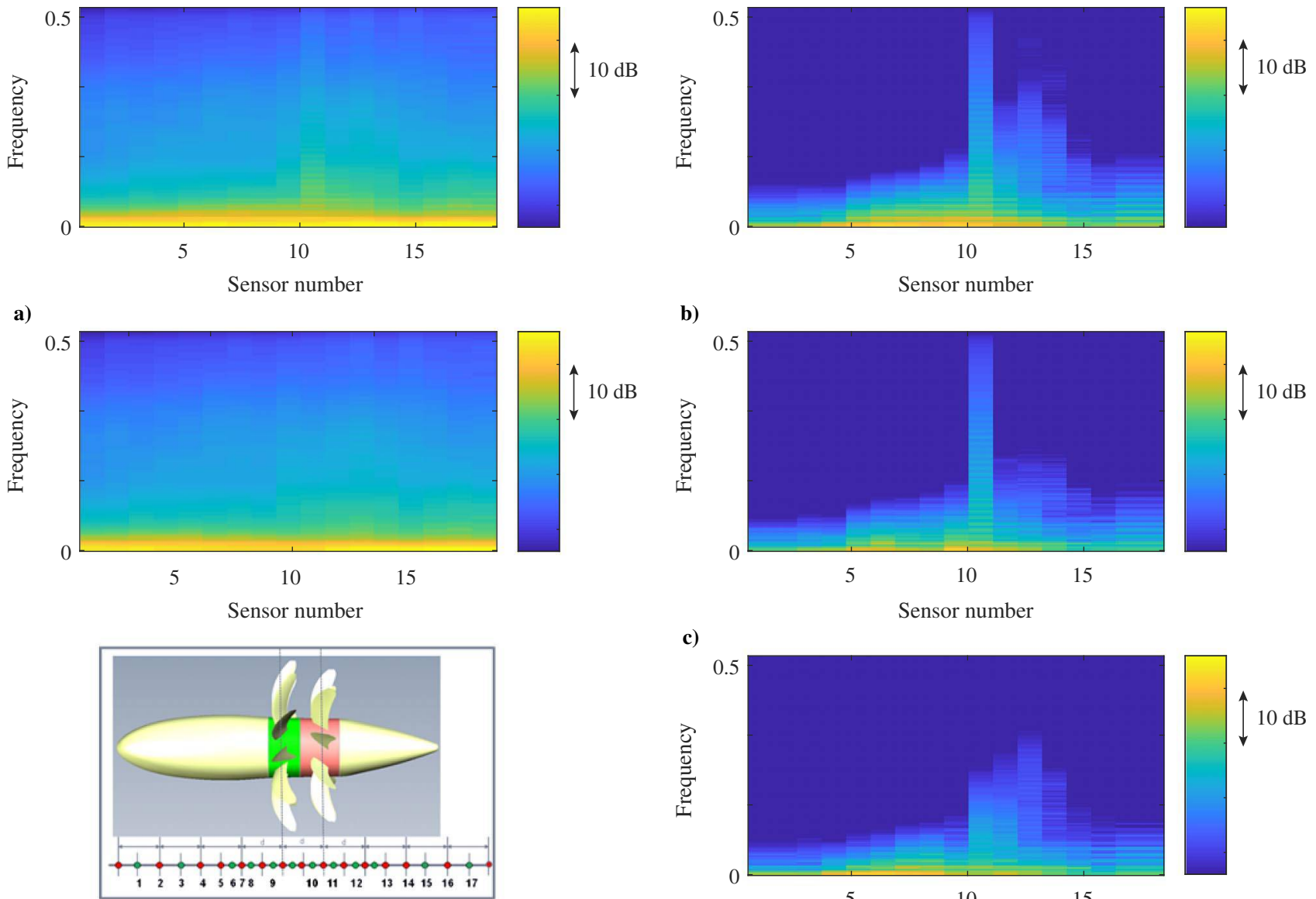

b)
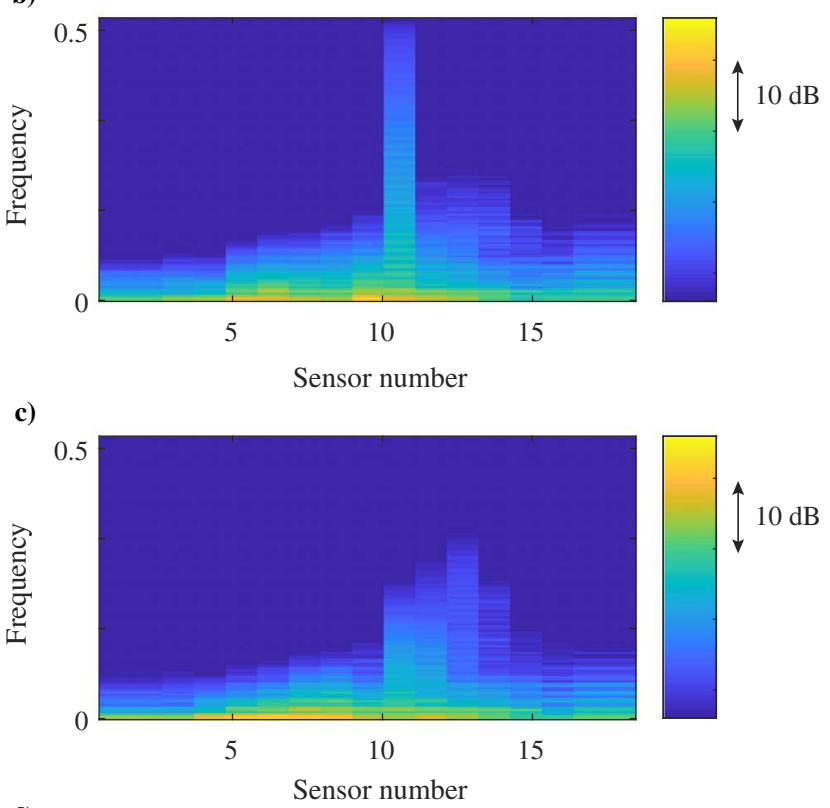

e)

d)

Fig. 13 Power spectral densities (arbitrary units) of AT-CS2 components as a function of frequency and space: a) total broadband part, b) AT-CS2 part due to rear and front rotors (including interactions), c) part due to front rotor only, d) part due to rear rotor only, and e) residual stationary noise $\left(\Delta f=4 \times 10^{-3} F_{s}\right)$.

interactions between the rotors, as indicated by Eq. (35). Finally, the residual of the decomposition amounts for stationary components (blue line). This is compared to the PSD of the noise reference measured in the wind tunnel, under the same flow conditions, with blades removed and without rotation of the rotors (blue dotted line).
Several observations are in order based on Figs. 11 and 12 and on Fig. 13, which collect the PSDs of the separated broadband parts for all sensors as a function of frequency and space.

The total power of the AT-CS2 components is not negligible as compared to that of the tonal component, an observation in 


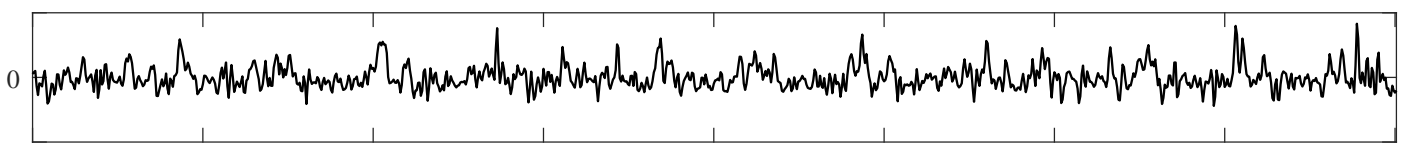

a)

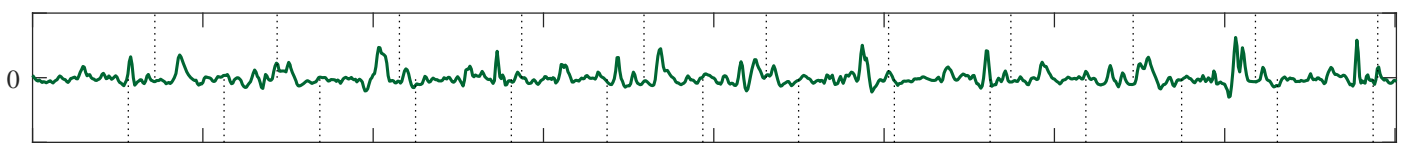

b)

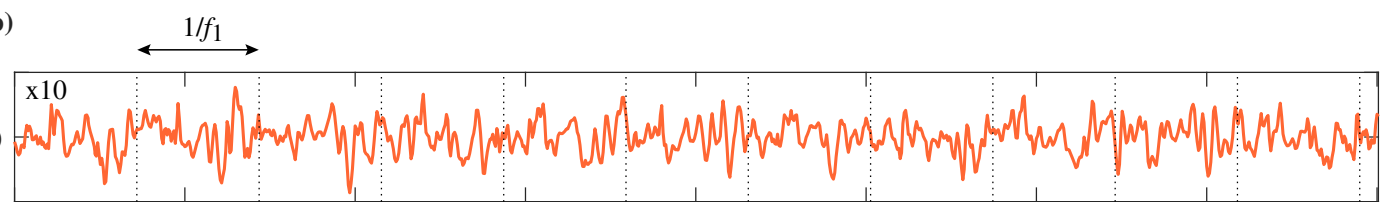

c)

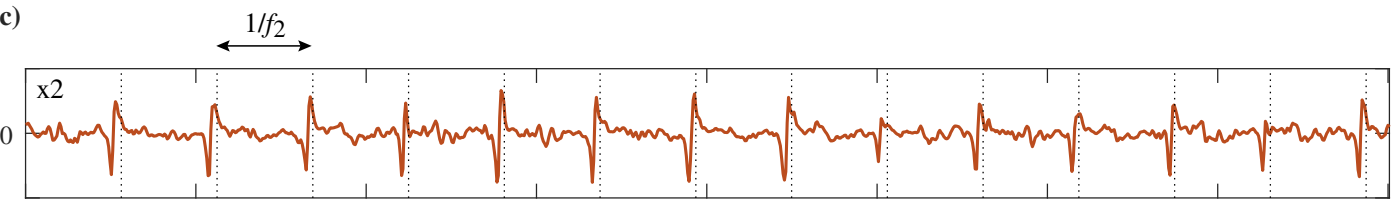

d)

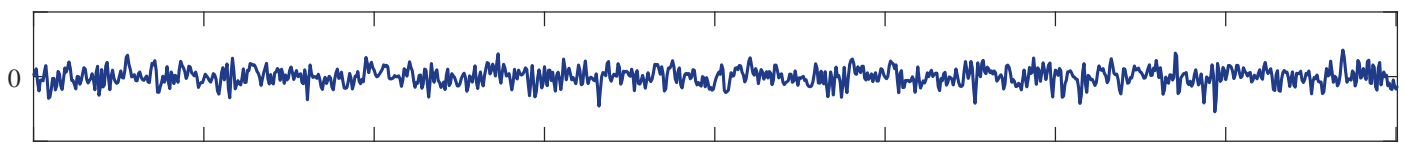

e)

Time

Fig. 14 Extracted time signals (time derivatives, arbitrary units) for sensor 11 with the same code of colors as in Figs. 11 and 12 : a) total broadband part, b) AT-CS2 part due to rear and front rotors (including interactions), c) AT-CS2 part due to front rotor only (note the change of scale), d) AT-CS2 part due to rear rotor only (note the change of scale), and e) residual stationary noise.

accordance with the literature on CROR [27]. On sensors with the smallest viewing angle with respect to the rotors, the two types of contributions are of comparable level; this is particularly present on sensor 10 (see Fig. 11), which is located right in front of the rotors. As for AT-CS2 components, it is seen in Fig. 13 that they are the strongest on sensors close to the planes that contain the rotors. Although an exact localization of the corresponding sources is yet not possible without further processing (e.g., with beamforming), it is likely that sensors 10 and 12 are in the main regions of radiation of the sources stemming from the front and rear rotors, respectively (accounting for the spatial shift due to the convection of waves) [28].

It is noteworthy that the total AT-CS2 part due to both rotors largely dominates the individual parts of each rotor; for some sensors and in some frequency ranges, the difference is greater than the $3 \mathrm{~dB}$ gap that would correspond to the simple addition of two independent contributions (e.g., on sensor 10, after $0.06 F_{s}$; see Fig. 11). According to Eq. (35), this indicates the presence of interactions between the two rotors, at frequencies $m f_{1}+n f_{2},(m, n) \in \mathbb{Z}^{2}$, especially in the high-frequency range, where the corresponding set of cyclic orders becomes very dense. The proposed cyclic regression scheme is well able to extract them, thanks to the high correlation they produce between frequency components.

Moreover, a close inspection of the PSD of the AT-CS2 components in Figs. 11 and 12 shows concentration of energy near groups of tones, even though the spectrum is truly continuous as opposed to the spectrum of tones. This reflects the presence of spreading of the tone power, likely due to modulation with turbulence (i.e., hay stacking and shear-layer effects), which could not be completely removed in the preliminary tonal extraction step. Whether these narrowband components actually pertain to the tonal part or to the "broadband" random part is an open question, as there exists a continuous transition from one to the other. What truly matters here is that they are correctly recovered in the form of AT-CS2 components if not completely removed with the tonal part. (It is reminded here that the aim of the study is primarily the extraction of contributions; an accurate localization of sources in space would require a subsequent analysis based on dedicated acoustic imaging techniques.)

Finally, the PSD of the residual stationary noise is seen to remain the dominant contribution above all. It follows quite closely the PSD of the reference noise, yet overestimates it by $3-10 \mathrm{~dB}$ above $0.016 F_{s}$. (The experimental configuration is here characterized by a high level of background noise due to the flow speed and to the presence of a reverberant environment.) The difference is expected to contain the residual stationary contributions of the CROR noise (i.e., the part not extracted as AT-CS2), yet due to the rotations of the rotors as explained in Sec. V, but also reflects irreducible mismatch between the experimental configurations with and without rotation of the rotors. [The turbulent boundary layer (TBL) level is probably higher when the CROR is operating.] Because the PSDs of the residual stationary parts are fairly constant in space (see Fig. 13), they are likely to be dominated by background noise due to the inflow and TBL.

Fig. 14 displays the separation results reconstructed in the time domain after application of the inverse STFT (9). The total broadband signal is compared to the AT-CS2 part due to two rotors, the front and rear rotors alone, and the stationary residual part. The time derivatives of the signals are shown in order to mitigate the strong contribution of the low-frequency components. The separated signals clearly reveal the AT-CS2 property imposed by the rotors; this takes the form of repetitive, but not periodic, waveforms, which tend to be quite impulsive on sensors close to the rotors. Sensor 11 shows that the strongest impulses are due to interactions between the two rotors.

\section{Conclusions}

A method has been proposed to decompose broadband noise into a cyclostationary part, typically attached to the operation of a rotor, and a stationary part related to background noise. As many cyclostationary contributions can be separated, as there are mechanical 
components rotating at different asynchronous speeds. Specific sources of noise can thus be extracted and analyzed individually as if they were measured alone with all other interfering sources of noise switched off. The method also makes possible the resynthesis of the signals in the time domain. Among other aims, this allows ranking sources according to their respective contributions to the global acoustical field. Because it is not based on a propagation model, the method does not aim at localizing sources in space; yet, it could be used as a preprocessing step before application of acoustic imaging techniques to separate sources, a topic that is left for future work. An important goal of this work is to illustrate the successive steps involved in the separation process, as well as provide guidelines as how to set up parameters in the algorithms. Criteria have been devised for allowing automatic selection of critical parameters, so as to relieve as much as possible the user intervention. The methodology has been illustrated on aeroacoustic signals recorded on a CROR engine in a wind tunnel. The broadband signals obtained after removing the tonal components have been further decomposed into cyclostationary contributions due to rotation of the rear and front propellers, their interactions, and the stationary background noise due to inflow and TBL. As far as the authors know, there is no equivalent decomposition in the literature.

\section{Appendix A: Model Selection}

The selection of the model order is here addressed as a test of hypothesis between two competing models. Let $\mathcal{M}_{k}$ denote a model with $k$ parameters arranged in a vector $\Theta_{k}$. Based on the observation of data $\boldsymbol{X} \in \mathbb{C}^{M \times I}$, the aim is to test whether the latter originate from model $\mathcal{M}_{k}$ or $\mathcal{M}_{k+1}$. This is akin to testing the two alternative hypotheses:

$$
\left\{\begin{array}{l}
H_{0}: X \sim p\left(\boldsymbol{X} \mid \mathcal{M}_{k}, \Theta_{k}\right) \\
H_{1}: X \sim p\left(\boldsymbol{X} \mid \mathcal{M}_{k+1}, \Theta_{k+1}\right)
\end{array}\right.
$$

where $p\left(\boldsymbol{X} \mid \mathcal{M}_{k}, \Theta_{k}\right)$ stands for the probability density function of $\boldsymbol{X}$ parameterized by $\Theta_{k}$ in model $\mathcal{M}_{k}$. Because the vector of parameters $\Theta_{k}$ is unknown, it is substituted by its maximum likelihood estimate $\hat{\Theta}_{k}$ [i.e., the vector that maximizes $\left.p\left(\boldsymbol{X} \mid \mathcal{M}_{k}, \Theta_{k}\right)\right]$. Thus, the decision is to reject the null hypothesis if the generalized likelihood ratio (GLR)

$$
\mathrm{GLR}=\ln \left(\frac{p\left(\boldsymbol{X} \mid \mathcal{M}_{k+1}, \hat{\Theta}_{k+1}\right)}{p\left(\boldsymbol{X} \mid \mathcal{M}_{k}, \hat{\Theta}_{k}\right)}\right)
$$

is found greater than a certain threshold. Assuming that $\boldsymbol{X}$ follows a multivariate complex Gaussian with mean $\sum_{l=1}^{k} \boldsymbol{C}_{l} \boldsymbol{X}_{l}$ and covariance matrix $\sigma^{2} \boldsymbol{I}$, one has

$$
\ln p\left(\boldsymbol{X} \mid \mathcal{M}_{k}, \Theta_{k}\right)=-\frac{\left\|\boldsymbol{X}-\sum_{l=1}^{k} \boldsymbol{C}_{l} \boldsymbol{X}_{l}\right\|^{2}}{\sigma^{2}}-I M \ln \sigma^{2}
$$

Replacing $\sigma^{2}$ by its maximum likelihood estimate $\hat{\sigma}^{2}$ given in Eq. (27), it comes

$$
\ln p\left(X \mid \mathcal{M}_{k}, \hat{\Theta}_{k}\right)=-I-I M \ln \hat{\sigma}^{2}=-I\left(1+M \ln \hat{\sigma}^{2}\right)
$$

Finally, substituting the latter result into Eq. (37) gives the lefthand side of Eq. (26). The right-hand side is obtained by invoking the result that, asymptotically

$$
-2 \ln \mathrm{GLR} \sim \chi_{\left|\Theta_{k+1}\right|-\left|\Theta_{k}\right|}^{2}
$$

with $\left|\Theta_{k}\right|$ the dimension of (i.e., the number of independent unknowns in) $\Theta$. As discussed in Sec. III.B.2, $\left|\Theta_{k}\right|=2 S(M(k+1)$ $-S)$. Therefore, $\left|\Theta_{k+1}\right|-\left|\Theta_{k}\right|=2 S M$.

The preceding results assume that all columns in $\boldsymbol{X}$ are statistically independent. In practice, this assumption is violated by the use of overlap in the short-time Fourier transform (STFT). Therefore, I should be replaced by the effective number of snapshots:

$$
\begin{aligned}
I_{\mathrm{eff}} & =\frac{I}{\rho(0)^{2}+2 \sum_{i=1}^{I-1}\left(1-\frac{i}{I}\right) \rho(i \Delta)^{2}} \leq I \quad \text { with } \\
\rho(i \Delta) & =\frac{\sum_{n} w\left(n / F_{s}\right) w\left((n-i \Delta) / F_{s}\right)}{\sum_{n} w^{2}\left(n / F_{s}\right)}
\end{aligned}
$$

which reflects the number of independent STFT coefficients in $\boldsymbol{X}$.

\section{Appendix B: Statistical Threshold of the Order- Frequency Spectral Coherence}

The statistical threshold (24) arrives as a particular case of the statistical test (37) when $M=1$ [i.e., $\boldsymbol{X}=\left[X_{m 1}(f), \ldots, X_{m I}(f)\right]$, $\left.\boldsymbol{X}_{1}=\left[X_{m 1}\left(f ; \alpha_{l}\right), \ldots, X_{m I}\left(f ; \alpha_{l}\right)\right]\right), \boldsymbol{C}_{1}=c_{1}$, and $k=1$. Therefore, $\Theta_{1}=\sigma_{0}^{2}, \Theta_{2}=\left(\sigma_{1}^{2}, c_{1}\right)$, and Eq. (A5) becomes $2 I_{\text {eff }} \ln \left(\hat{\sigma}_{1}^{2} / \hat{\sigma}_{0}^{2}\right) \sim \chi_{2}^{2}$. The last step is to recognize that $\hat{\gamma}^{2}\left(\alpha_{l}, f\right)=1-\hat{\sigma}_{1}^{2} / \hat{\sigma}_{0}^{2}$, from which Eq. (24) immediately follows.

\section{Acknowledgments}

This work was funded by Clean Sky 2 Joint Undertaking, European Union, Horizon 2020, CS2-RIA, ADAPT project, grant agreement number 754881 . This work was partly performed within the Labex CeLyA of the Universite de Lyon, in the program 'Investissements d'Avenir' (ANR-10-LABX-0060/ANR-16-IDEX-0005) operated by the French National Research Agency (Agence Nationale de la Recherche).

\section{References}

[1] Stephens, D. B., and Vold, H., "Order Tracking Signal Processing for Open Rotor Acoustics," Journal of Sound and Vibration, Vol. 333, No. 16, 2014, pp. 3818-3830. https://doi.org/10.1016/j.jsv.2014.04.005

[2] Sree, D., and Stephens, D., "Improved Separation of Tone and Broadband Noise Components from Open Rotor Acoustic Data," Aerospace, Vol. 3, No. 29, 2016, p. 29.

[3] Antoni, J., Leclère, Q., Dinsenmeyer, A., Julliard, E., Bouley, S., Picard, C., and Sijtsma, P., "Standalone Extraction of Tonal Components from Aeroacoustic Signals," AIAA Journal (to be published).

[4] Truong, A., and Papamoschou, D., "Harmonic and Broadband Separation of Noise from a Small Ducted Fan," 21 st AIAA/CEAS Aeroacoustics Conference, AIAA Paper 2015-3282, June 2015. https://doi.org/10.2514/6.2015-3282

[5] Behn, M., Pardowitz, B., and Tapken, U., "Separation of Tonal and Broadband Noise Components by Cyclostationary Analysis of the Modal Sound Field in a Low-Speed Fan Test Rig," International Conference of Fan Noise, Aerodynamics, Applications and Systems, 2018.

[6] Tokaji, K., and Horváth, C., "Combining Signal Pre-Processing Methods with Beamforming for Broadband Turbomachinery Applications," 7th Berlin Beamforming Conference (BeBeC), 2018.

[7] Tokaji, K., Soós, B., and Horváth, C., "Beamforming Method for Extracting the Broadband Noise Sources of Counter-Rotating Open Rotors," AIAA Journal, Vol. 58, No. 7, 2020, pp. 3028-3039. https://doi.org/10.2514/1.J058934

[8] Magliozzi, B., Hanson, D. B., and Amiet, R. K., "Aeroacoustics of Flight Vehicles: Theory and Practice. Volume 1: Noise Sources," NASA TR 2, 1991.

[9] Glegg, S., and Devenport, W., "Open Rotor Noise," Aeroacoustics of Low Mach Number Flows, edited by S. Glegg, and W. Devenport, Academic Press, New York, 2017, pp. 399-436. https://doi.org/10.1016/B978-0-12-809651-2.00016-3

[10] Enghardt, L., Moreau, A., Tapken, U., and Kennepohl, F., "Radial Mode Decomposition in the Outlet of a LPT Turbine-Estimation of the Relative Importance of Broadband Noise," 15th AIAA/CEAS Aeroacoustics Conference, AIAA Paper 2009-3286, May 2009, https://elib .dlr.de/62864/.

[11] Horváth, C., "Beamforming Investigation of Dominant CounterRotating Open Rotor Tonal and Broadband Noise Sources," AIAA Journal, Vol. 53, No. 6, 2015, pp. 1602-1611. https://doi.org/10.2514/1.J053465 
[12] Hurd, H. L., "Nonparametric Time Series Analysis for Periodically Correlated Processes," IEEE Transactions on Information Theory, Vol. 35, No. 2, 1989, pp. 350-359.

[13] Antoni, J., "Cyclostationarity by Examples," Mechanical Systems and Signal Processing, Vol. 23, No. 4, 2009, pp. 987-1036. https://doi.org/10.1016/j.ymssp.2008.10.010

[14] George, V. V., Gaonkar, G. H., Prasad, J. V. R., and Schrage, D. P., "Adequacy of Modeling Turbulence and Related Effects on Helicopter Response," AIAA Journal, Vol. 30, No. 6, 1992, pp. 1468-1479. https://doi.org/10.2514/3.11088

[15] VanZante, D., Feddersen, R., Suarez, M., and Sherman, P., "The Stochastic Structure of Downstream Pressure from an Axial Compressor-I. A Periodic Time-Frequency Spectral Description," Mechanical Systems and Signal Processing, Vol. 10, No. 4, 1996, pp. 413-422 https://doi.org/10.1006/mssp.1996.0029

[16] Sherman, P., Dudley, R., and Suarez, M., "The Stochastic Structure of Downstream Pressure from an Axial Compressor-II. An Investigation of Blade-to-Blade Variability," Mechanical Systems and Signal Processing, Vol. 10, No. 4, 1996, pp. 423-437. https://doi.org/10.1006/mssp.1996.0030

[17] Jurdic, V., Joseph, P., and Antoni, J., "Investigation of Rotor Wake Turbulence Through Cyclostationary Spectral Analysis," AIAA Journal, Vol. 47, No. 9, 2009, pp. 2022-2030. https://doi.org/10.2514/1.36728

[18] Cheong, C., and Joseph, P., "Cyclostationary Spectral Analysis for the Measurement and Prediction of Wind Turbine Swishing Noise," Journal of Sound and Vibration, Vol. 333, No. 14, 2014, pp. 3153-3176. https://doi.org/10.1016/j.jsv.2014.02.031

[19] Abboud, D., and Antoni, J., "Order-Frequency Analysis of Machine Signals," Mechanical Systems and Signal Processing, Vol. 87, March 2017, pp. 229-258. https://doi.org/10.1016/i.ymssp.2016.10.024

[20] Graham, W. R., "Cross-Correlation-Based Identification of Rotating Sources," AIAA Journal, Vol. 57, No. 8, 2019, pp. 3456-3466. https://doi.org/10.2514/1.J058016
[21] Yu, L., Antoni, J., Wu, H., and Jiang, W., "Reconstruction of Cyclostationary Sound Source Based on a Back-Propagating Cyclic Wiener Filter," Journal of Sound and Vibration, Vol. 442, March 2019, pp. 787-799. https://doi.org/10.1016/j.jsv.2018.11.034

[22] Yu, L., Wu, H., Antoni, J., and Jiang, W., "Extraction and Imaging of Aerodynamically Generated Sound Field of Rotor Blades in the Wind Tunnel Test," Mechanical Systems and Signal Processing, Vol. 116, Feb. 2019, pp. 1017-1028. https://doi.org/10.1016/j.ymssp.2018.07.042

[23] Brillinger, D. R., Time Series: Data Analysis and Theory, Soc. for Industrial and Applied Mathematics, New York, 1975, Chap. 4.

[24] Boustany, R., and Antoni, J., "Blind Extraction of a Cyclostationary Signal Using Reduced-Rank Cyclic Regression-A Unifying Approach," Mechanical Systems and Signal Processing, Vol. 22, No. 3, 2008, pp. 520-541. https://doi.org/10.1016/j.ymssp.2007.09.014

[25] Antoni, J., Bonnardot, F., Raad, A., and Badaoui, M. E., "Cyclostationary Modelling of Rotating Machine Vibration Signals," Mechanical Systems and Signal Processing, Vol. 18, No. 6, 2004, pp. 1285-1314. https://doi.org/10.1016/S0888-3270(03)00088-8

[26] Markovsky, I., "Structured Low-Rank Approximation and Its Applications," Automatica, Vol. 44, No. 4, 2008, pp. 891-909. https://doi.org/10.1016/j.automatica.2007.09.011

[27] Parry, A., Kingan, M., and Tester, B., "Relative Importance of Open Rotortone and Broadband Noise Sources," 17th AIAA/CEAS Aeroacoustics Conference (32nd AIAA Aeroacoustics Conference), AIAA Paper 2011-2763, June 2011. https://doi.org/10.2514/6.2011-2763

[28] Blandeau, V. P., and Joseph, P. F., "Broadband Noise Due to RotorWake/Rotor Interaction in Contra-Rotating Open Rotors," AIAA Journal, Vol. 48, No. 11, 2010, pp. 2674-2686. https://doi.org/10.2514/1.J050566

D. Papamoschou Associate Editor 